\title{
On extensions of the core and the anticore of transferable utility games
}

Citation for published version (APA):

Derks, J., Peters, H. J. M., \& Sudhölter, P. (2012). On extensions of the core and the anticore of transferable utility games. METEOR, Maastricht University School of Business and Economics. METEOR Research Memorandum No. 003 https://doi.org/10.26481/umamet.2012003

Document status and date:

Published: 01/01/2012

DOI:

10.26481/umamet.2012003

Document Version:

Publisher's PDF, also known as Version of record

\section{Please check the document version of this publication:}

- A submitted manuscript is the version of the article upon submission and before peer-review. There can be important differences between the submitted version and the official published version of record.

People interested in the research are advised to contact the author for the final version of the publication, or visit the DOI to the publisher's website.

- The final author version and the galley proof are versions of the publication after peer review.

- The final published version features the final layout of the paper including the volume, issue and page numbers.

Link to publication

\footnotetext{
General rights rights.

- You may freely distribute the URL identifying the publication in the public portal. please follow below link for the End User Agreement:

www.umlib.nl/taverne-license

Take down policy

If you believe that this document breaches copyright please contact us at:

repository@maastrichtuniversity.nl

providing details and we will investigate your claim.
}

Copyright and moral rights for the publications made accessible in the public portal are retained by the authors and/or other copyright owners and it is a condition of accessing publications that users recognise and abide by the legal requirements associated with these

- Users may download and print one copy of any publication from the public portal for the purpose of private study or research.

- You may not further distribute the material or use it for any profit-making activity or commercial gain

If the publication is distributed under the terms of Article $25 \mathrm{fa}$ of the Dutch Copyright Act, indicated by the "Taverne" license above, 


\title{
On extensions of the core and the anticore of transferable utility games*
}

\author{
Jean Derks ${ }^{\dagger} \quad$ Hans Peters ${ }^{\ddagger} \quad$ Peter Sudhölter $^{\S}$
}

This version, January 2012

\begin{abstract}
We consider several related set extensions of the core and the anticore of games with transferable utility. An efficient allocation is undominated if it cannot be improved, in a specific way, by sidepayments changing the allocation or the game. The set of all such allocations is called the undominated set, and we show that it consists of finitely many polytopes with a core-like structure. One of these polytopes is the $L_{1}$-center, consisting of all efficient allocations that minimize the sum of the absolute values of the excesses. The excess Pareto optimal set contains the allocations that are Pareto optimal in the set obtained by ordering the sums of the absolute values of the excesses of coalitions and the absolute values of the excesses of their complements. The $L_{1}$-center is contained in the excess Pareto optimal set, which in turn is contained in the undominated set. For three-person games all these sets coincide. These three sets also coincide with the core for balanced games and with the anticore for antibalanced games. We study properties of these sets and provide characterizations in terms of balanced collections of coalitions. We also propose a singlevalued selection from the excess Pareto optimal set, the min-prenucleolus, which is defined as the prenucleolus of the minimum of a game and its dual.
\end{abstract}

Keywords: Transferable utility game, core, anticore, core extension, minprenucleolus

JEL classification: C71

AMS classification: $91 \mathrm{~A} 12$

* Thanks are due to Elena Yanovskaya for helpful comments. The third author was supported by the Spanish Ministerio de Ciencia y Innovación under project ECO2009-11213, co-funded by ERDF.

${ }^{\dagger}$ Department of Knowledge Engineering, Maastricht University, e-mail address: jean.derks@maastrichtuniversity.nl.

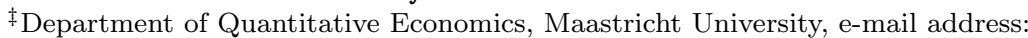
h.peters@maastrichtuniversity.nl.

$\S$ Department of Business and Economics, University of Southern Denmark, e-mail address: psu@sam.sdu.dk. 


\section{Introduction}

The core of a game with transferable utility can be interpreted from a strategic point of view - a coalition may 'deviate' from the grand coalition if it obtains less than its stand-alone worth - but also from a fairness point of view: if one coalition obtains its worth or more, then it is only fair that all coalitions obtain at least their worths. From the fairness point of view, a natural extension of the core for games in which the core is empty, would be the anticore, provided that this is a nonempty set. The anticore is the set of efficient allocations where every coalition obtains at most its worth, and the fairness argument now says that, if one coalition obtains its worth or less, then it is only fair that all coalitions obtain at most their worths. In general, however, both the core and the anticore of a game may be empty, and then the question is what a 'natural' extension of the core or the anticore for such games could be.

Of course, this question is not new. A classical extension of the core is the $\varepsilon$ core, based on subtracting $\varepsilon$ from the coalitional worths, and the least core, i.e., the intersection of all nonempty $\varepsilon$-cores (Shapley and Shubik, 1966; Maschler et al, 1979). Recently, Bejan and Gómez (2009) propose, alternatively, to increase the worth of the grand coalition to obtain a nonempty core, where this increase is financed by taxing individual players. The resulting solution concept is called the extended core. All such extensions have in common that, essentially, the core restrictions are relaxed in order to obtain a nonempty solution. Our approach is quite different, and there are no obvious relations between the mentioned extensions (least core, extended core) and our extensions.

We start by defining undominated efficient allocations. An efficient allocation $x$ is undominated in a game $v$ if there is no other efficient allocation $z$ and no game $w$ arising from sidepayments between players (i.e., by adding an additive game to $v$ ) such that for each coalition $S$ the minimum of $z(S)$ (its total payoff from $z$ ) and $w(S)$ is at least as large as, and sometimes strictly larger than, the minimum of $x(S)$ and $v(S)$; the allocation $z$ and the game $w$ are interpreted as arising from bargaining over $x$ and $v$, respectively, and considering the minimum is based on an assumption of (pessimistic) max-min preferences concerning uncertainty of reaching a final agreement $(x$ or $z)$ or not $(v$ or $w)$. The set of all undominated efficient allocations is called the undominated set, and we show, indeed, that for balanced games this set coincides with the core and for antibalanced games with the anticore. We characterize the undominated set by balancedness conditions. These conditions imply that being undominated is equivalent to the impossibility of redistributing the payoffs among all coalitions with nonpositive excesses such that no coalition with negative excess loses, and redistributing the payoffs among all coalitions with nonnegative excesses such that no coalition with positive excess loses. In fact, this characterization can serve as an intuitive alternative definition, and in a specific sense reflects the fairness consideration discussed earlier.

We propose two other set extensions of the core and the anticore. The $L_{1}$ center, first introduced in Spinetto (1974), consists of all efficient allocations that minimize the sum of the absolute values of the coalitional excesses. The 
excess Pareto optimal set consists of all efficient allocations that are associated with Pareto optimal points (with the understanding that lower values are better) of the set of vectors arising from computing, for each coalition, the sum of the absolute value of its excess and the absolute value of the excess of its complement, over all possible efficient allocations. The $L_{1}$-center is contained in the excess Pareto optimal set, which in turn is contained in the undominated set. The latter two coincide on the interesting class of proper games (i.e., games in which the worth of a coalition plus the worth of its complement is never larger than the worth of the grand coalition). All three sets coincide with the core for balanced games and the anticore for antibalanced games. They also coincide for all three-person games. The undominated set is the union of a finite collection of polytopes, each with a core-like structure. The $L_{1}$-center is exactly one of these polytopes, and the excess Pareto optimal set is the union of a specific subcollection of these polytopes.

Besides studying further properties of these three set extensions we also propose a single-valued solution which selects from the excess Pareto optimal set and thus from the undominated set. This solution is a modification of the prenucleolus (Schmeidler, 1969), called the min-prenucleolus, and it assigns to a game the prenucleolus of the coalition-wise minimum of that game and its dual. We present an axiomatic characterization, based on the characterization of the prenucleolus of Sobolev (1975).

The paper is organized as follows. After preliminaries in Section 2 we introduce the undominated set in Section 3, and characterize it by balanced collections and relate it to the core and the anticore in Section 4 . The $L_{1}$-center and the excess Pareto optimal set are discussed in Sections 5 and 6 , respectively. In Section 7 we show that the excess Pareto optimal set is contractible. Section 8 is devoted to the min-prenucleolus and its characterization, while Section 9 briefly reconsiders the undominated set for proper games. Section 10 concludes.

\section{Preliminaries}

A game with transferable utility or, simply, a game is a pair $(N, v)$, where $N=\{1, \ldots, n\}$ with $n \in \mathbb{N}$ is the set of players and the function $v: 2^{N} \rightarrow \mathbb{R}$ with $v(\emptyset)=0$ is the characteristic function, assigning to each coalition $S \subseteq N$ its worth $v(S)$. We also call $(N, v)$ an $n$-person game and often write $v$ instead of $(N, v)$ if there is no confusion about the player set. We sometimes write $i j k \ldots$ for a coalition $\{i, j, k, \ldots\} \subseteq N$.

An ( $n$-person) allocation is a vector $x \in \mathbb{R}^{N}$. For an allocation $x$ and a coalition $S \subseteq N$ we denote $x(S):=\sum_{i \in S} x_{i}$, with the convention $x(\emptyset):=0$. An allocation $x$ is efficient in the game $v$ if $x(N)=v(N)$. The set of efficient allocations in $v$ is denoted by $X(v)$.

A game $v$ is additive if $v(S \cup T)=v(S)+v(T)$ for all $S, T \in 2^{N}$ such that $S \cap T=\emptyset$. Note that such a game is completely described by the worths of the singleton coalitions. In particular, any allocation $x$ can be identified with an additive game $v^{x}$ by letting $v^{x}(\{i\})=x_{i}$ for all $i \in N$; in this case we usually 
write $x$ instead of $v^{x}$. The sum $v+w$ of two games $v$ and $w$ is defined by $(v+w)(S)=v(S)+w(S)$ for all $S \in 2^{N}$. In particular, for $x \in \mathbb{R}^{N}, v+x$ is the sum of $v$ and the additive game induced by $x$.

A collection of coalitions $\mathcal{B} \subseteq 2^{N}$ is balanced if there are positive numbers $\lambda(S), S \in \mathcal{B}$, such that $\sum_{S \in \mathcal{B}: i \in S} \lambda(S)=1$ for every $i \in N$. The numbers $\lambda(S)$ are called balancing weights.

A sidepayment for the player set $N$ is a vector $y \in \mathbb{R}^{N}$ with $y(N)=0$.

A game $v$ is balanced [antibalanced] if $\sum_{S \in \mathcal{B}} \lambda(S) v(S) \leqslant[\geqslant] v(N)$ for every balanced collection $\mathcal{B}$, with balancing weights $\lambda(S)$. Clearly, an additive game is both balanced and antibalanced, but any other game is either balanced or antibalanced or none of the two. The core $C(v)$ of a game $v$ is the set $\{x \in X(v) \mid$ $x(S) \geqslant v(S)$ for all $\left.S \in 2^{N}\right\}$ and the anticore $A C(v)$ is the set $\{x \in X(v)$ $x(S) \leqslant v(S)$ for all $\left.S \in 2^{N}\right\}$. It is well-known (Bondareva, 1962; Shapley, 1967) that $C(v) \neq \emptyset$ if and only if $v$ is balanced, and similarly that $A C(v) \neq \emptyset$ if and only if $v$ is antibalanced.

Sidepayments and balanced collections can be related as follows. For $\mathcal{B} \subseteq$ $\mathcal{B}^{\prime} \subseteq 2^{N}$, we say that $\mathcal{B}$ is balanced within $\mathcal{B}^{\prime}$ if there exists a balanced collection $\mathcal{B}^{\prime \prime}$ with $\mathcal{B} \subseteq \mathcal{B}^{\prime \prime} \subseteq \mathcal{B}^{\prime}$. Then we have the following useful result.

Lemma 2.1 A collection $\mathcal{B}$ is balanced within a collection $\mathcal{B}^{\prime}$ if and only if for each sidepayment $y \in \mathbb{R}^{N}$ with $y(S) \geqslant 0$ for all $S \in \mathcal{B}^{\prime}$ we have $y(S)=0$ for all $S \in \mathcal{B}$. In particular, a collection $\mathcal{B}$ is balanced if and only if for each sidepayment $y \in \mathbb{R}^{N}$ with $y(S) \geqslant 0$ for all $S \in \mathcal{B}$ we have $y(S)=0$ for all $S \in \mathcal{B}$.

A proof of this lemma, based on Farkas' Lemma, can be found in Derks and Peters (1998).

\section{The undominated set}

Our definition of the undominated set of a game $v$ will be based on a new concept of domination among efficient allocations. In order to motivate this new concept, consider a game $v$ and an efficient allocation $x \in X(v)$. Assume that the proposal $x$ is on the table, and consider a coalition $S$. From the point of view of $S$, if the final decision is exclusively about $x$, then $S$ is going to obtain $x(S)$ if an agreement on $x$ is reached, and $v(S)$ otherwise, hence $S$ is sure to obtain the minimum of $x(S)$ and $v(S)$. This reflects a coalition having max-min preferences over the uncertain issue of reaching agreement or not. However, consider the following two possibilities before the actual agreement or disagreement decision on a proposal is taken. First, $x$ may be replaced by another efficient allocation $z \in X(v)$; equivalently, $x$ is replaced by $x+y$, where $y$ is a sidepayment. Second, players may make prepayments among each other, effectively turning the game $v$ into a new game $v+y^{\prime}$, where $y^{\prime}$ is the additive game induced by these prepayments, i.e., $y^{\prime}$ is again a sidepayment. After such a bargaining phase, from the point of view of coalition $S$, if an agreement will be reached then $S$ is going to obtain $(x+y)(S)$, and otherwise it is going to 
obtain $\left(v+y^{\prime}\right)(S)$; hence, $S$ is sure to obtain the minimum of $(x+y)(S)$ and $\left(v+y^{\prime}\right)(S)$.

With this setting in mind, we present the following definition.

Definition 3.1 Let $v$ be a game and let $x \in X(v)$. Then $x$ is dominated if there are sidepayments $y$ and $y^{\prime}$ such that

$$
\min \left\{(x+y)(S),\left(v+y^{\prime}\right)(S)\right\} \geqslant \min \{x(S), v(S)\} \text { for all } S \in 2^{N}
$$

with at least one of these inequalities strict. If $x$ is not dominated, then it is undominated. The set of all undominated effcient allocations is the undominated set, denoted by $U D(v)$.

Thus, if $x \in X(v)$ is undominated, then there are no sidepayments $y, y^{\prime}$ such that all coalitions are better off and at least one coalition is strictly better off by deciding on the allocation $x+y$ instead of $x$ and at the same time performing sidepayments $y^{\prime}$. Observe, again, that 'better off' is based, implicitly, on the coalitions having max-min preferences to deal with the uncertainty of the players reaching an agreement on a proposal.

Remark 3.2 This concept of domination is closely related to the dominance relation between pairs of allocations considered in Bossert, Derks and Peters (2005) in the context of uncertain cooperative games.

\section{Characterization of the undominated set by balanced collections}

The undominated set can be characterized in terms of balanced collections. This yields a finite check on (un)dominatedness of an efficient allocation, and is convenient for computational purposes. Moreover, the characterization provides relations with the core and the anticore of a game. More generally, it is useful for investigating the geometric structure of the undominated set. Specifically, we will show that the undominated set is a union of finitely many polytopes.

We start with an auxiliary lemma on extending balanced collections.

Lemma 4.1 Let $\mathcal{B}_{1}$ and $\mathcal{B}_{2}$ be balanced collections in $2^{N}$. Then there are balanced collections $\mathcal{C}_{1}$ and $\mathcal{C}_{2}$ such that

(1) $\mathcal{B}_{1} \subseteq \mathcal{C}_{1} \subseteq \mathcal{B}_{1} \cup\left(2^{N} \backslash \mathcal{B}_{2}\right)$,

(2) $\mathcal{B}_{2} \subseteq \mathcal{C}_{2} \subseteq \mathcal{B}_{2} \cup\left(2^{N} \backslash \mathcal{B}_{1}\right)$,

(3) $\mathcal{C}_{1} \cup \mathcal{C}_{2}=2^{N}$

Proof. Suppose that $S \in 2^{N}$ such that $S \notin \mathcal{B}_{1} \cup \mathcal{B}_{2}$. If $N \backslash S \in \mathcal{B}_{1}$ then $\{S\} \cup \mathcal{B}_{1}$ is still balanced; if $N \backslash S \in \mathcal{B}_{2}$ then $\{S\} \cup \mathcal{B}_{2}$ is still balanced; and if $N \backslash S \notin \mathcal{B}_{1} \cup \mathcal{B}_{2}$ then $\{S, N \backslash S\} \cup \mathcal{B}_{1}$ (or $\left.\{S, N \backslash S\} \cup \mathcal{B}_{2}\right)$ is still balanced. 
Thus, $S$ or $S$ and $N \backslash S$ can be added to $\mathcal{B}_{1}$ or $\mathcal{B}_{2}$. The desired sets $\mathcal{C}_{1}$ and $\mathcal{C}_{2}$ are obtained by repeating this argument.

In order to state the characterization theorem, for a game $v$ and an efficient allocation $x \in X(v)$ we define the following collections of coalitions:

$$
\begin{aligned}
& L(x, v)=\left\{S \in 2^{N} \mid x(S)<v(S)\right\} \\
& E(x, v)=\left\{S \in 2^{N} \mid x(S)=v(S)\right\} \\
& H(x, v)=\left\{S \in 2^{N} \mid x(S)>v(S)\right\} .
\end{aligned}
$$

When there is no confusion about what the game is, we often write $L(x), E(x)$, and $H(x)$ instead of $L(x, v), E(x, v)$, and $H(x, v)$.

Theorem 4.2 Let $v$ be a game and let $x \in X(v)$. Then $x$ is undominated if and only if there are balanced collections $\mathcal{B}_{1}$ and $\mathcal{B}_{2}$ such that $L(x) \subseteq \mathcal{B}_{1}$, $H(x) \subseteq \mathcal{B}_{2}$, and $\mathcal{B}_{1} \cap \mathcal{B}_{2} \subseteq E(x)$.

Proof. For the only-if part, let $x$ be undominated. We show that $L(x)$ is balanced within $L(x) \cup E(x)$. Suppose not, then according to Lemma 2.1 there is a sidepayment $y$ with $y(S) \geqslant 0$ for all $S \in L(x) \cup E(x)$ and $y(S)>0$ for some $S \in L(x)$. Choose $\alpha>0$ such that $x(S)+\alpha y(S)>v(S)$ for all $S \in H(x)$. Then we have

$$
\min \{x(S)+\alpha y(S), v(S)\} \geqslant \min \{x(S), v(S)\} \text { for all } S \in 2^{N}
$$

with at least one inequality strict, as is easy to verify. This violates undominatedness of $x$. Hence $L(x)$ is balanced within $L(x) \cup E(x)$. Similarly, one shows that $H(x)$ is balanced within $H(x) \cup E(x)$. So we have shown that there are balanced collections $\mathcal{B}_{1}$ and $\mathcal{B}_{2}$ with $L(x) \subseteq \mathcal{B}_{1} \subseteq L(x) \cup E(x)$ and $H(x) \subseteq \mathcal{B}_{2} \subseteq H(x) \cup E(x)$.

For the if-part, let $\mathcal{B}_{1}$ and $\mathcal{B}_{2}$ be as in the statement of the theorem. In view of Lemma 4.1 we may assume that $\mathcal{B}_{1} \cup \mathcal{B}_{2}=2^{N}$. Suppose there are sidepayments $y$ and $y^{\prime}$ such that

$$
\min \left\{x(S)+y(S), v(S)+y^{\prime}(S)\right\} \geqslant \min \{x(S), v(S)\} \text { for all } S \in 2^{N} .
$$

For $S \in L(x) \cup E(x),(1)$ implies $\min \left\{x(S)+y(S), v(S)+y^{\prime}(S)\right\} \geqslant x(S)$, which in turn implies $y(S) \geqslant 0$. Since $\mathcal{B}_{1}$ is balanced it is also balanced within $L(x) \cup$ $E(x)$, which by Lemma 2.1 implies $y(S)=0$ for all $S \in \mathcal{B}_{1}$. Similarly, one shows that $y^{\prime}(S)=0$ for all $S \in \mathcal{B}_{2}$. Since $\mathcal{B}_{1} \cup \mathcal{B}_{2}=2^{N}$, it follows that all inequalities in (1) are equalities, so that $x$ is undominated.

The following corollary follows from Theorem 4.2 and Lemma 4.1.

Corollary 4.3 Let $v$ be a game and let $x \in X(v)$. Then $x$ is undominated if and only if there are balanced collections $\mathcal{B}_{1}$ and $\mathcal{B}_{2}$ such that $L(x) \subseteq \mathcal{B}_{1}$, $H(x) \subseteq \mathcal{B}_{2}, \mathcal{B}_{1} \cap \mathcal{B}_{2} \subseteq E(x)$, and $\mathcal{B}_{1} \cup \mathcal{B}_{2}=2^{N}$. 
Lemma 2.1 and Theorem 4.2 (or Corollary 4.3) provide a further explanation of undominated allocations and, indeed, could be used as alternative definitions. Define the excess of a coalition $S$ in a game $v$ at an efficient allocation $x$ by $e(S, x, v)=e(S, x)=v(S)-x(S)$. Then an efficient allocation is undominated exactly if by any sidepayment (redistribution) that makes no coalition with nonpositive excess worse off no coalition with negative excess can strictly improve, and by any sidepayment that makes no coalition with nonnegative excess worse off no coalition with positive excess can strictly improve.

As an illustration, in the next example we compute the undominated set for three-person symmetric games (already considered in von Neumann and Morgenstern, 1944).

Example 4.4 Let $N=\{1,2,3\}$, let $\alpha \in \mathbb{R}^{N}$, and consider the three-person symmetric game $v$ with $v(i)=0$ for all $i \in N, v(i j)=\alpha$ for all $i, j \in N$ with $i \neq j$, and $v(N)=1$. For $\alpha \leqslant \frac{2}{3}$ we have $U D(v)=C(v)=\left\{x \in \mathbb{R}^{3} \mid\right.$ $x_{i} \geqslant 0$ for all $i \in N, x(N)=1$, and $x_{i}+x_{j} \geqslant \alpha$ for all $\left.i, j \in N, i \neq j\right\}$. For $1>$ $\alpha>\frac{2}{3}$ we have $C(v)=\emptyset$ and $U D(v)=\left\{x \in \mathbb{R}^{3} \mid x_{i} \geqslant 0\right.$ for all $i \in N, x(N)=1$, and $x_{i}+x_{j} \leqslant \alpha$ for all $\left.i, j \in N, i \neq j\right\}$. For $\alpha \geqslant 1$ we have $U D(v)=\left\{x \in \mathbb{R}^{3} \mid\right.$ $x(N)=1$ and $x_{i} \geqslant 0$ for all $\left.i \in N\right\}$. All these statements can be checked by using Theorem 4.2 or Corollary 4.3.

The next result shows that the undominated set extends the core and the anticore.

Theorem 4.5 Let $v$ be a game. If $C(v) \neq \emptyset$ then $U D(v)=C(v)$ and if $A C(v) \neq$ $\emptyset$ then $U D(v)=A C(v)$.

Proof. Suppose that $C(v) \neq \emptyset$, and let $z \in C(v)$. Since $E(z) \cup H(z)=2^{N}$ and $2^{N}$ is balanced, Theorem 4.2 implies that $z$ is undominated, so $C(v) \subseteq U D(v)$. To prove the converse inclusion, let $\tilde{x}$ be an arbitrary element of $C(v)$. Then for every $S \in 2^{N}$ and every $x \in X(v)$ we have $\min \{x(S)+(\tilde{x}-x)(S), v(S)\}=$ $v(S) \geqslant \min \{x(S), v(S)\}$. If, in particular, $x$ is undominated then we must have $\min \{x(S)+(\tilde{x}-x)(S), v(S)\}=v(S)=\min \{x(S), v(S)\}$ for all $S \in 2^{N}$, hence $x(S) \geqslant v(S)$ for all $S \in 2^{N}$. So $U D(v) \subseteq C(v)$. This proves the first implication in the theorem. The proof of the second implication is analogous ${ }^{1}$.

The following example shows that in general the undominated set does not have to be convex.

Example 4.6 Consider the four-person game $v$ with worths given in the following table.

$\begin{array}{cccccccccccccccc}S & 1 & 2 & 3 & 4 & 12 & 13 & 14 & 23 & 24 & 34 & 123 & 124 & 134 & 234 & N \\ v(S) & -5 & -5 & -2 & 5 & 5 & -5 & 5 & 5 & -5 & 5 & -1 & -5 & -5 & -5 & 0\end{array}$

Then $x=(-2,-2,2,2) \in U D(v)$ (check the balancedness conditions in Theorem $4.2)$ and so is $y=-x$. However, $\frac{x+y}{2}=0 \notin U D(v): E(0)=\{\emptyset, N\}$, and

\footnotetext{
${ }^{1}$ Alternatively, one can use the first implication together with the observations $U D(v)=$ $U D\left(v^{*}\right)$ and $A C(v)=C\left(v^{*}\right)$, where $v^{*}$ is the dual game of $v$, see Sections 6 and 8 below.
} 
$L(0)=\{12,23,34,14,4\}$, which cannot be extended to a balanced collection by adding elements from $E(0)$. So $U D(v)$ is not convex.

We now further explore the relation between the undominated set, the core and the anticore. For a game $v$ and an arbitrary collection $\mathcal{B} \subseteq 2^{N}$ define the $\mathcal{B}$-restricted core and anticore by

$$
C(\mathcal{B}, v)=\{x \in X(v) \mid x(S) \geqslant v(S) \text { for all } S \in \mathcal{B}\}
$$

and

$$
A C(\mathcal{B}, v)=\{x \in X(v) \mid x(S) \leqslant v(S) \text { for all } S \in \mathcal{B}\} .
$$

Let $\mathcal{P}=\mathcal{P}^{N}$ denote the set of all pairs of balanced collections with player set $N$ such that all coalitions are used, i.e.,

$$
\mathcal{P}=\left\{\left(\mathcal{B}_{1}, \mathcal{B}_{2}\right) \subseteq 2^{N} \times 2^{N} \mid \mathcal{B}_{1}, \mathcal{B}_{2} \text { are balanced and } \mathcal{B}_{1} \cup \mathcal{B}_{2}=2^{N}\right\}
$$

An element of $\mathcal{P}$ is called a constellation.

We now have:

Theorem 4.7 Let $v$ be a game. Then

$$
U D(v)=\bigcup_{\left(\mathcal{B}_{1}, \mathcal{B}_{2}\right) \in \mathcal{P}} A C\left(\mathcal{B}_{1}, v\right) \cap C\left(\mathcal{B}_{2}, v\right) .
$$

Proof. If $x \in U D(v)$ then by Corollary 4.3 there are balanced collections $\mathcal{B}_{1}$ and $\mathcal{B}_{2}$ with $L(x) \subseteq \mathcal{B}_{1} \subseteq L(x) \cup E(x), H(x) \subseteq \mathcal{B}_{2} \subseteq H(x) \cup E(x)$, and $\mathcal{B}_{1} \cup \mathcal{B}_{2}=2^{N}$, and clearly $x \in A C\left(\mathcal{B}_{1}, v\right) \cap C\left(\mathcal{B}_{2}, v\right)$. Conversely, let $x \in X(v)$ and $\left(\mathcal{B}_{1}, \mathcal{B}_{2}\right) \in \mathcal{P}$ such that $x \in A C\left(\mathcal{B}_{1}, v\right) \cap C\left(\mathcal{B}_{2}, v\right)$. Then $L(x) \subseteq \mathcal{B}_{1} \subseteq L(x) \cup E(x)$ and $H(x) \subseteq \mathcal{B}_{2} \subseteq H(x) \cup E(x)$ with both collections balanced, so $x \in U D(v)$ by Theorem 4.2.

Theorem 4.7 says that the undominated set is the union of finitely many polyhedra, each one with a core-like structure. We can actually say more.

Lemma 4.8 Let $v$ be a game and let $\left(\mathcal{B}_{1}, \mathcal{B}_{2}\right) \in \mathcal{P}$. Then the set $A C\left(\mathcal{B}_{1}, v\right) \cap$ $C\left(\mathcal{B}_{2}, v\right)$ is compact.

Proof. Closedness is obvious. Suppose the set were not bounded. Then there must be a player $i \in N$ such that, for every number $K \in \mathbb{R}$ there is an $x \in$ $A C\left(\mathcal{B}_{1}, v\right) \cap C\left(\mathcal{B}_{2}, v\right)$ with $x_{i} \geqslant K$. Clearly, $\{i\} \in \mathcal{B}_{2}$. Since $\mathcal{B}_{2}$ is balanced we have $\sum_{S \in \mathcal{B}_{2}} \lambda(S) x(S)=x(N)=v(N)$ for every $x \in X(v)$, where $\lambda(S)>0$, $S \in \mathcal{B}_{2}$, are balancing weights associated with $\mathcal{B}_{2}$. By choosing $x \in A C\left(\mathcal{B}_{1}, v\right) \cap$ $C\left(\mathcal{B}_{2}, v\right)$ with $x(\{i\})=x_{i}$ large enough, there must be a coalition $T \in \mathcal{B}_{2}$ with $x(T)<v(T)$, contradicting the fact that $x \in C\left(\mathcal{B}_{2}, v\right)$. Hence the set $A C\left(\mathcal{B}_{1}, v\right) \cap C\left(\mathcal{B}_{2}, v\right)$ must be bounded.

Lemma 4.8 and Theorem 4.7 imply that the undominated set is the union of finitely many polytopes. In particular, we have the following consequence. 
Corollary 4.9 For every game $v$ the set $U D(v)$ is compact.

Many of the polytopes in Theorem 4.7 can be empty. For instance, if $v$ is a balanced game, then for the only nonempty polytope, the core, we can take the constellation $\mathcal{P}=\left(\mathcal{B}_{1}, \mathcal{B}_{2}\right)$ where $\mathcal{B}_{1}=\{\emptyset, N\}$ (so that $A C\left(\mathcal{B}_{1}, v\right)=X(v)$ ) and $\mathcal{B}_{2}=2^{N}$ (so that $C\left(\mathcal{B}_{2}, v\right)=C(v)$ ), as follows from Theorem 4.5. Nonemptiness of the undominated set for arbitrary $v$ will follow as a result in the next section (Corollary 5.4).

In general, different pairs $\left(\mathcal{B}_{1}, \mathcal{B}_{2}\right) \in \mathcal{P}$ may lead to the same set $A C\left(\mathcal{B}_{1}, v\right) \cap$ $C\left(\mathcal{B}_{2}, v\right)$. In order to achieve a canonical representation, call $\left(\mathcal{B}_{1}, \mathcal{B}_{2}\right) \in \mathcal{P}$ maximal with respect to the game $v$ if there exists an $x \in A C\left(\mathcal{B}_{1}, v\right) \cap C\left(\mathcal{B}_{2}, v\right)$ such that

$$
L(x)=\mathcal{B}_{1} \backslash \mathcal{B}_{2} \text { and } H(x)=\mathcal{B}_{2} \backslash \mathcal{B}_{1} .
$$

The following lemma shows that we indeed obtain a canonical, 'unique' representation of $U D(v)$ based on maximal constellations.

Lemma 4.10 Let $v$ be a game and let $\mathcal{P}_{v}^{m}$ denote the set of maximal constellations with respect to $v$. Then

$$
U D(v)=\bigcup_{\left(\mathcal{B}_{1}, \mathcal{B}_{2}\right) \in \mathcal{P}_{v}^{m}} A C\left(\mathcal{B}_{1}, v\right) \cap C\left(\mathcal{B}_{2}, v\right)
$$

and

$$
U D(v) \supseteq \bigcup_{\left(\mathcal{B}_{1}, \mathcal{B}_{2}\right) \in \mathcal{P}_{v}^{m} \backslash\left\{\left(\widehat{\mathcal{B}}_{1}, \widehat{\mathcal{B}}_{2}\right)\right\}} A C\left(\mathcal{B}_{1}, v\right) \cap C\left(\mathcal{B}_{2}, v\right) \text { for all }\left(\widehat{\mathcal{B}}_{1}, \widehat{\mathcal{B}}_{2}\right) \in \mathcal{P}_{v}^{m} .
$$

Proof. Clearly, an allocation $x$ as in (2) for a maximal constellation does not belong to the polytope of any other maximal constellation with respect to $v$. Together with Theorem 4.7 this implies (4). Also one inclusion of (3) follows from Theorem 4.7. In order to show the other inclusion let $z \in U D(v)$ and take $\left(\mathcal{B}_{1}, \mathcal{B}_{2}\right) \in \mathcal{P}$ such that $z \in P:=A C\left(\mathcal{B}_{1}, v\right) \cap C\left(\mathcal{B}_{2}, v\right)$. Define the sets $L, E$, and $H$ by

$$
\begin{aligned}
L & =\left\{S \in 2^{N} \mid S \in L(x) \text { for some } x \in P\right\} \\
E & =\left\{S \in 2^{N} \mid S \in E(x) \text { for all } x \in P\right\} \\
H & =\left\{S \in 2^{N} \mid S \in H(x) \text { for some } x \in P\right\} .
\end{aligned}
$$

Let $\widehat{\mathcal{B}}_{1}=L \cup E$ and $\widehat{\mathcal{B}}_{2}=H \cup E$. By convexity of $P$ there is an $\hat{x} \in P$ such that $L(\hat{x})=L=\widehat{\mathcal{B}}_{1} \backslash \widehat{\mathcal{B}}_{2}$ and $H(\hat{x})=H=\widehat{\mathcal{B}}_{2} \backslash \widehat{\mathcal{B}}_{1}$. Also, $P=A C\left(\widehat{\mathcal{B}}_{1}\right) \cap C\left(\widehat{\mathcal{B}}_{2}\right)$, so it is sufficient to prove that $\widehat{\mathcal{B}}_{1}$ and $\widehat{\mathcal{B}}_{2}$ are balanced. Consider a sidepayment $y \in \mathbb{R}^{N}$ with $y(S) \geqslant 0$ for all $S \in \widehat{\mathcal{B}}_{1}=L \cup E$. We may assume that $(\hat{x}+y)(S)>v(S)$ for all $S \in H$. Since $L \subseteq \mathcal{B}_{1} \subseteq L \cup E$ and $\mathcal{B}_{1}$ is balanced, we have $y(S)=0$ for all $S \in \mathcal{B}_{1}$ by Lemma 2.1. Then we must have $y(S)=0$ for all $S \in E$ as well, since otherwise $\hat{x}+y$ would be an element of $P$ with $(\hat{x}+y)(S)>v(S)$ for some $S \in E$, contradicting the definition of $E$. Hence, $y(S)=0$ for all $S \in L \cup E$, implying balancedness of $\widehat{\mathcal{B}}_{1}=L \cup E$ by Lemma 2.1 . One similarly proves that $\widehat{\mathcal{B}}_{2}$ is balanced. 
Remark 4.11 Lemma 4.10 says that the undominated set of a game $v$ consists of a collection of distinct polytopes which correspond one-to-one with the maximal constellations with respect to $v$. The proof of the lemma in fact shows that any nonempty polytope generated by some arbitrary possibly nonmaximal constellation is equal to one of these polytopes.

Note, further, that if $\left(\mathcal{B}_{1}, \mathcal{B}_{2}\right) \in \mathcal{P}$ is maximal with respect to $v$ then the dimension of the set $A C\left(\mathcal{B}_{1}, v\right) \cap C\left(\mathcal{B}_{2}, v\right)$ - i.e., the dimension of the smallest affine subspace of $\mathbb{R}^{n}$ containing it - is equal to $n-\left|\mathcal{B}_{1} \cap \mathcal{B}_{2}\right|+1$, hence maximally $n-1 .^{2}$

We denote the relative interior of a set $Z \subseteq \mathbb{R}^{n}$ - i.e., the interior of $Z$ relative to a smallest affine subspace of $\mathbb{R}^{n}$ containing $Z$ - by $\operatorname{relint}(Z)$. Now we have:

Lemma 4.12 Let the constellation $\left(\mathcal{B}_{1}, \mathcal{B}_{2}\right) \in \mathcal{P}$ be maximal with respect to the game $v$. Then for every $x \in X(v)$ we have $x \in \operatorname{relint}\left(A C\left(\mathcal{B}_{1}, v\right) \cap C\left(\mathcal{B}_{2}, v\right)\right)$ if and only if $L(x) \cup E(x)=\mathcal{B}_{1}$ and $H(x) \cup E(x)=\mathcal{B}_{2}$. In particular, if $x \in \operatorname{relint}\left(A C\left(\mathcal{B}_{1}, v\right) \cap C\left(\mathcal{B}_{2}, v\right)\right)$, then $L(x) \cup E(x)$ and $H(x) \cup E(x)$ are balanced.

Proof. Let $x \in X(v)$. Then $x \in \operatorname{relint}\left(A C\left(\mathcal{B}_{1}, v\right) \cap C\left(\mathcal{B}_{2}, v\right)\right)$ if and only if $x(S)<v(S)$ for all $S \in \mathcal{B}_{1} \backslash \mathcal{B}_{2}, x(S)>v(S)$ for all $S \in \mathcal{B}_{2} \backslash \mathcal{B}_{1}$, and $x(S)=v(S)$ for all $S \in \mathcal{B}_{1} \cap \mathcal{B}_{2}$. Hence, $x \in \operatorname{relint}\left(A C\left(\mathcal{B}_{1}, v\right) \cap C\left(\mathcal{B}_{2}, v\right)\right)$ if and only if $L(x) \cup E(x)=\mathcal{B}_{1}$ and $H(x) \cup E(x)=\mathcal{B}_{2}$. The last claim in the lemma is obvious.

In a game $v$, the sets $L(x) \cup E(x)$ and $H(x) \cup E(x)$ for an undominated allocation $x \in X(v)$ are not necessarily balanced themselves. Lemmas 4.10 and 4.12 however imply that these sets are balanced if $x$ is a relative interior allocation of the polytope to which it belongs. Hence, such allocations are elements of the set $s U D(v)$ defined by

$$
s U D(v)=\{x \in X(v) \mid L(x) \cup E(x) \text { and } H(x) \cup E(x) \text { are balanced }\} .
$$

Thus we have

$$
\bigcup_{\left(\mathcal{B}_{1}, \mathcal{B}_{2}\right) \in \mathcal{P}} \operatorname{relint}\left(A C\left(\mathcal{B}_{1}, v\right) \cap C\left(\mathcal{B}_{2}, v\right)\right) \subseteq s U D(v) \subseteq U D(v) .
$$

Since for every $\left(\mathcal{B}_{1}, \mathcal{B}_{2}\right) \in \mathcal{P}$ the set $A C\left(\mathcal{B}_{1}, v\right) \cap C\left(\mathcal{B}_{2}, v\right)$ is the (topological) closure of relint $\left(A C\left(\mathcal{B}_{1}, v\right) \cap C\left(\mathcal{B}_{2}, v\right)\right)$, it follows that the set at the left-hand side in $(5)$ and thus also the set $s U D(v)$ is dense in $U D(v)$. The following example shows that both inclusions can be strict.

Example 4.13 Let $(N, v)$ be defined by $N=\{1,2,3\}, v(12)=v(3)=1$, $v(13)=v(1)=-1$ and $v(S)=0$ for all other $S \subseteq N$. Then the undominated set is the convex hull of the set $\{(0,0,0),(-1,0,1),(-1,1,0),(0,1,-1)\}$. For

${ }^{2}$ For a set $D$, we denote its cardinality by $|D|$. 
$x=(0,0,0)$ we have $L(x)=\{3,12\}, E(x)=\{\emptyset, 2,23, N\}$, and $H(x)=\{1,13\}$ so that $x \in U D(v) \backslash s U D(v)$. For $z=(-1,0,1)$ we have $L(z)=\{12\}, E(z)=$ $\{\emptyset, 1,2,3, N\}$, and $H(z)=\{13,23\}$, so that $z \in s U D(v) \backslash \operatorname{relint}(U D(v))$. This example may be extended to more than three players by adding null-players.

Remark 4.14 Yanovskaya (2002) introduces the 'extended prenucleolus' of a game $v$, which we denote by $Y(v)$. Theorem 1 in Yanovskaya (1998) states that $x \in X(v)$ belongs to $Y(v)$ if and only if the collection $L(x) \cup E(x)$ is balanced. For balanced games, $Y(v)$ coincides with the relative interior of the core, and hence is a strict subset of the undominated set. In general, there is no inclusion relation: the condition on $Y(v)$ is weaker in the sense that there is no balancedness condition involving $H(x)$, but stronger in the sense that $L(x) \cup E(x)$ is required to be balanced instead of $L(x)$ within $L(x) \cup E(x)$, as in the undominated set. For an example, consider the three-person game $v^{\prime}$ with $v^{\prime}(12)=2$ and $v^{\prime}(S)=v(S)$ for all other coalitions $S$, with $v$ as in Example 4.13. Then for $x=(0,0,0)$ we have $L\left(x, v^{\prime}\right)=\{3,12\}, E\left(x, v^{\prime}\right)=\{\emptyset, 2,23, N\}$, and $H\left(x, v^{\prime}\right)=\{1,13\}$ so that $x \in U D(v) \backslash Y(v)$. For $z=\left(\frac{1}{3}, \frac{4}{3},-\frac{5}{3}\right)$ we have $L\left(z, v^{\prime}\right)=\{3,12,13,23\}, E\left(z, v^{\prime}\right)=\{\emptyset, N\}$, and $H\left(z, v^{\prime}\right)=\{1,2\}$, so that $z \in Y(v) \backslash U D(v)$.

\section{The $L_{1}$-center}

The $L_{1}$-center of a game $v$ was introduced by Spinetto (1974), who showed that it coincides with the core for balanced games. We will see that it is also closely related to the undominated set. More precisely, it is exactly one of the polytopes of which the undominated set consists.

Definition 5.1 For a game $v$, let the function $\ell: \mathbb{R}^{N} \rightarrow \mathbb{R}$ be defined by

$$
\ell(x)=\sum_{S \in 2^{N}}|v(S)-x(S)| .
$$

The $L_{1}$-center of the game $v$ is the set

$$
L_{1}(v)=\left\{x \in X(v) \mid \ell(x) \leqslant \ell\left(x^{\prime}\right) \text { for all } x^{\prime} \in X(v)\right\} .
$$

Thus, the $L_{1}$-center consists of all efficient allocations that minimize the sum of the absolute values of the coalitional excesses. Since $f$ is a continuous function and we can restrict ourselves to a bounded and closed subset of $X(v)$ to find its minima, it follows by the extreme value theorem of Weierstraß that the $L_{1}$-center is nonempty; it is also clear that it is closed and therefore compact. By using the triangular inequality for absolute values it is easy to see that the $L_{1}$-center is a convex set. Altogether we have the following result.

Lemma 5.2 Let $v$ be a game. Then $L_{1}(v)$ is nonempty, compact and convex.

The next result shows that the $L_{1}$-center coincides with exactly one of the polytopes in Theorem 4.7 . 
Theorem 5.3 Let $v$ be a game. Then there is a pair $\left(\mathcal{B}_{1}, \mathcal{B}_{2}\right) \in \mathcal{P}$ such that $L_{1}(v)=A C\left(\mathcal{B}_{1}, v\right) \cap C\left(\mathcal{B}_{2}, v\right)$.

Proof. We first prove the following two claims.

Claim 1 If $x, x^{\prime} \in L_{1}(v)$ and $S \in L(x)$, then $S \notin H\left(x^{\prime}\right)$.

To show this, suppose to the contrary that $x, x^{\prime} \in L_{1}(v), S \in L(x)$, and $S \in H\left(x^{\prime}\right)$. Let $0<t<1$ and consider the efficient allocation $z=t x+(1-t) x^{\prime}$. Then $|v(S)-z(S)|=\left|t(v(S)-x(S))+(1-t)\left(v(S)-x^{\prime}(S)\right)\right|<t|v(S)-x(S)|+(1-$ t) $\left|v(S)-x^{\prime}(S)\right|$ since $v(S)-x(S)>0$ and $v(S)-x^{\prime}(S)<0$. Also, for any $T \in 2^{N}$, we have $|v(T)-z(T)| \leqslant t|v(T)-x(T)|+(1-t)\left|v(T)-x^{\prime}(T)\right|$ by the triangular inequality. Hence, $\ell(z)<t \ell(x)+(1-t) \ell\left(x^{\prime}\right)=\min \left\{\ell\left(x^{\prime \prime}\right) \mid x^{\prime \prime} \in X(v)\right\}$, contradicting the fact that $z \in X(v)$. This proves Claim 1 .

Claim 2 Let $x \in L_{1}(v)$. If $y$ is a sidepayment with $y(S) \geqslant 0$ for all $S \in E(x)$, then $\sum_{S \in L(x)} y(S) \leqslant 0$ and $\sum_{S \in H(x)} y(S) \geqslant 0$.

To show this, let $y$ be a sidepayment with $y(S) \geqslant 0$ for all $S \in E(x)$. Let $\varepsilon>0$ be so small that $L(x+\varepsilon y)=L(x), H(x+\varepsilon y) \supseteq H(x), L(x-\varepsilon y) \supseteq L(x)$, and $H(x-\varepsilon y)=H(x)$. Then

$$
\begin{aligned}
\ell(x+\varepsilon y)= & \sum_{S \in L(x+\varepsilon y)}(v(S)-x(S)-\varepsilon y(S)) \\
& +\sum_{S \in H(x+\varepsilon y)}(x(S)+\varepsilon y(S)-v(S)) \\
= & \sum_{S \in L(x)}(v(S)-x(S))-\varepsilon \sum_{S \in L(x)} y(S) \\
& +\sum_{S \in H(x)}(x(S)-v(S))+\varepsilon \sum_{S \in H(x)} y(S)+\varepsilon \sum_{S \in E(x)} y(S) \\
= & \ell(x)-2 \varepsilon \sum_{S \in L(x)} y(S) .
\end{aligned}
$$

This implies $\sum_{S \in L(x)} y(S) \leqslant 0$ since $x \in L_{1}(v)$. One similarly shows

$$
\ell(x-\varepsilon y)=\ell(x)+2 \varepsilon \sum_{S \in H(x)} y(S)
$$

which implies $\sum_{S \in H(x)} y(S) \geqslant 0$. This proves Claim 2.

We now define $L=\left\{S \in 2^{N} \mid S \in L(x)\right.$ for some $\left.x \in L_{1}(v)\right\}$ and $H=$ $\left\{S \in 2^{N} \mid S \in H(x)\right.$ for some $\left.x \in L_{1}(v)\right\}$. For each $S \in L$ take an $x^{S} \in$ $L_{1}(v)$ such that $S \in L\left(x^{S}\right)$ and for each $S \in H$ take an $x^{S} \in L_{1}(v)$ such that $S \in H\left(x^{S}\right)$. Let $\hat{x}$ be a convex combination of all these $x^{S}$ with positive weights. By convexity of the $L_{1}$-center (Lemma 5.2) we have $\hat{x} \in L_{1}$ and by Claim $1, L=L(\hat{x})$ and $H=H(\hat{x})$. Writing $E=E(\hat{x})=2^{N} \backslash(L \cup H)$ (so $E \subseteq E(x)$ for all $x \in L_{1}(v)$ ), we claim that $L \cup E$ and $H \cup E$ are balanced. Let $y$ be a sidepayment with $y(S) \geqslant 0$ for all $S \in L \cup E$. By Claim 2 we have $\sum_{S \in H(\hat{x})} y(S) \geqslant 0$, hence $\sum_{S \in L \cup E} y(S) \leqslant 0$ (since $\sum_{S \in 2^{N}} y(S)=0$ ), so that $y(S)=0$ for all $S \in L \cup E$. Lemma 2.1 implies that $L \cup E$ is balanced. 
Similarly one shows that $H \cup E$ is balanced. Thus, $(L \cup E, H \cup E) \in \mathcal{P}$ and clearly $L_{1}(v) \subseteq A C(L \cup E, v) \cap C(H \cup E, v) .^{3}$

For the converse inclusion $L_{1}(v) \supseteq A C(L \cup E, v) \cap C(H \cup E, v)$, let $z \in$ $A C(L \cup E, v) \cap C(H \cup E, v)$. If $z(S)<v(S)$ for some $S \in 2^{N}$ then $S \in L$ and thus $\hat{x}(S)<v(S)$. If $z(S)>v(S)$ for some $S \in 2^{N}$ then $S \in H$ and thus $\hat{x}(S)>v(S)$. Hence, if $\hat{x}(S)=v(S)$ for some $S$ then also $z(S)=v(S)$. Define the sidepayment $y$ by $z=\hat{x}+y$, then $y(S)=0$ for all $S \in E$. By Claim 2 it follows that $\ell(z)=\ell(\hat{x})$, so that $z \in L_{1}(v)$. Thus, $L_{1}(v) \supseteq A C(L \cup E, v) \cap C(H \cup E, v)$.

An immediate consequence of Theorems 5.3 and 4.7 and Lemma 5.2 is nonemptiness of the undominated set.

Corollary 5.4 $L_{1}(v) \subseteq U D(v)$ and in particular $U D(v) \neq \emptyset$ for every game $v$.

The following result shows that for three-person games the $L_{1}$-center and the undominated set coincide.

Theorem 5.5 Let $v$ be a three-person game. Then $L_{1}(v)=U D(v)$.

The proof of this theorem is based on an extensive case distinction, and deferred to the appendix of this paper.

For games with more than three players the $L_{1}$-center can be a strict subset of the undominated set. This follows from the fact that the undominated set is not necessarily convex, as was shown by Example 4.6.

\section{Excess Pareto optimal allocations}

The $L_{1}$-center of a game $v$ contains the efficient allocations that minimize the sum of the absolute values of the excesses. In this section we further study the relation between these excesses and the undominated set. For a game $v$, a coalition $S \subseteq N$, and an allocation $x \in X(v)$ we define

$$
f(S, x, v)=f(S, x)=|v(S)-x(S)|+|v(N \backslash S)-x(N \backslash S)|
$$

and $f(x, v)=f(x)=(f(S, x))_{S \subseteq N} \in \mathbb{R}^{2^{N}}$. We also define the set $D(x, v)=$ $D(x)$ by

$$
D(x)=\left\{x^{\prime} \in X(v) \mid f\left(x^{\prime}\right) \leqslant f(x), f\left(x^{\prime}\right) \neq f(x)\right\} .
$$

Definition 6.1 Let $v$ be a game and $x \in X(v)$. Then $x$ is excess Pareto optimal if $D(x)=\emptyset$. The set of excess Pareto optimal allocations is denoted by $E P(v)$.

The amount $f(S, x)$ is the total absolute excess of the coalition $S$ and its complement at $x$. If $x \in X(v)$ is excess Pareto optimal, then there is no efficient allocation that has all these amounts lower, and at least one of them strictly

\footnotetext{
${ }^{3}$ Observe that $(L \cup E, H \cup E)$ is a maximal constellation. Its construction is similar to the one in the proof of Lemma 4.10. Cf. also Remark 4.11
} 
lower. ${ }^{4}$ Since $\ell(x)=\frac{1}{2} \sum_{S \subset N} f(S, x)$, and $\ell(x)$ is minimal exactly on $L_{1}(v)$, it follows that $L_{1}(v) \subseteq E P(v)$. Below we show that $E P(v)$ is a subset of $U D(v)$, but we start by characterizing $E P(v)$ by balanced collections. For a game $v$ and for $x \in X(v)$ we define the following collections of sets:

$$
L H(x, v)=L H(x)=\{S \in L(x) \mid N \backslash S \in H(x)\}
$$

and

$$
\operatorname{LEH}(x, v)=\operatorname{LEH}(x)=\{S \in L(x) \cup E(x) \mid N \backslash S \in H(x) \cup E(x)\} .
$$

Clearly, $L H(x) \subseteq L E H(x)$.

The following lemma characterizes excess Pareto optimal allocations in terms of sidepayments.

Lemma 6.2 Let $v$ be a game and let $x \in X(v)$. Then $x \in E P(v)$ if and only if for every sidepayment $y \in \mathbb{R}^{N}$ with $y(S) \geqslant 0$ for all $S \in L E H(x)$ we have $y(S)=0$ for all $S \in L H(x)$.

Proof. We will use the following claim, the straightforward proof of which is left to the reader.

Claim Let $y$ be a sidepayment. Then there is an $\varepsilon>0$ with $f(x+\varepsilon y) \leqslant f(x)$ if and only if $y(S) \geqslant 0$ for all $S \in L E H(x)$.

For the only-if direction of the lemma, let $x \in E P(v)$ and let $y \in \mathbb{R}^{N}$ be a sidepayment with $y(S) \geqslant 0$ for all $S \in L E H(x)$. Then by the Claim there is an $\varepsilon>0$ such that $f(x+\varepsilon y) \leqslant f(x)$. Since $x \in E P(v)$ this implies $f(x+\varepsilon y)=f(x)$. In particular, consider $S \in L H(x)$. Then $f(S, x+\varepsilon y)=f(S, x)$ implies

$$
\begin{gathered}
v(S)-x(S)-\varepsilon y(S)+x(N \backslash S)+\varepsilon y(N \backslash S)-v(N \backslash S) \\
=v(S)-x(S)+x(N \backslash S)-v(N \backslash S)
\end{gathered}
$$

which in turn implies $\varepsilon(y(N \backslash S)-y(S))=0$, hence $\varepsilon(-2 y(S))=0$ and thus $y(S)=0$.

For the if-direction assume that the sidepayment condition in the lemma holds for $x$. Let $z \in X(v)$ such that $f(z) \leqslant f(x)$. Then by the Claim the sidepayment $y=z-x$ satisfies $y(S) \geqslant 0$ for all $S \in L E H(x)$, so that $y(S)=0$ for all $S \in L H(x)$. Therefore, if $S \in L H(x)$, then $f(S, z)=f(S, x)$, and if $N \backslash S \in L H(x)$, then again $f(S, z)=f(S, x)$. Also, if $S, N \backslash S \in L(x) \cup E(x)$ or $S, N \backslash S \in H(x) \cup E(x)$, then $f(S, z) \leqslant f(S, x)$ implies $f(S, z)=f(S, x)$, since in those cases $f(S, x)$ is minimal over all efficient allocations. Hence, $f(z)=f(x)$, so that $z \notin D(x)$. Since $z$ was arbitrary we conclude that $x \in E P(v)$.

Lemmas 2.1 and 6.2 now imply:

Theorem 6.3 Let $v$ be a game and let $x \in X(v)$. Then $x \in E P(v)$ if and only if $L H(x)$ is balanced within $\operatorname{LEH}(x)$.

\footnotetext{
${ }^{4}$ Hence, Pareto optimality means 'Pareto minimality' here.
} 
We will use this characterization of $E P(v)$ to show that it consists of a subset of the finitely many polytopes that constitute $U D(v)$. Define the subset $\mathcal{P}^{c}$ of $\mathcal{P}$ as follows. A constellation $\left(\mathcal{B}_{1}, \mathcal{B}_{2}\right)$ is in $\mathcal{P}^{c}$ if the set

$$
\left\{S \in \mathcal{B}_{1} \mid N \backslash S \in \mathcal{B}_{2}\right\}
$$

is balanced.

Theorem 6.4 Let $v$ be a game. Then

$$
E P(v)=\bigcup_{\left(\mathcal{B}_{1}, \mathcal{B}_{2}\right) \in \mathcal{P}^{c}} A C\left(\mathcal{B}_{1}, v\right) \cap C\left(\mathcal{B}_{2}, v\right)
$$

Proof. First suppose that $\left(\mathcal{B}_{1}, \mathcal{B}_{2}\right) \in \mathcal{P}^{c}$ and $x \in A C\left(\mathcal{B}_{1}, v\right) \cap C\left(\mathcal{B}_{2}, v\right)$. Let $\mathcal{B}=\left\{S \in \mathcal{B}_{1} \mid N \backslash S \in \mathcal{B}_{2}\right\}$. Then $L H(x) \subseteq \mathcal{B} \subseteq L E H(x)$. Since $\mathcal{B}$ is balanced, this implies $x \in E P(v)$ by Theorem 6.3.

Conversely, suppose that $x \in E P(v)$. Then by Theorem 6.3 there is a balanced collection $\mathcal{B}$ with $L H(x) \subseteq \mathcal{B} \subseteq L E H(x)$. Define $\mathcal{B}_{1}=\{\emptyset, N\} \cup$ $\mathcal{B} \cup\{S, N \backslash S \mid S \in L(x), N \backslash S \in L(x) \cup E(x)\}$ and $\mathcal{B}_{2}=\{\emptyset, N\} \cup\{S \in$ $\left.2^{N} \mid N \backslash S \in \mathcal{B}\right\} \cup\left\{S, N \backslash S \in 2^{N} \mid S, N \backslash S \in E(x) \cup H(x)\right\}$. Then $\left\{S \in \mathcal{B}_{1} \mid N \backslash S \in \mathcal{B}_{2}\right\}=\mathcal{B} \cup\{\emptyset, N\}$ is balanced, $\mathcal{B}_{1}$ and $\mathcal{B}_{2}$ are balanced, and $\mathcal{B}_{1} \cup \mathcal{B}_{2}=2^{N}$, so $\left(\mathcal{B}_{1}, \mathcal{B}_{2}\right) \in \mathcal{P}^{c}$. Moreover, $x \in A C\left(\mathcal{B}_{1}, v\right) \cap C\left(\mathcal{B}_{2}, v\right)$. This proves the converse inclusion in the theorem.

Theorems 6.4 and 4.7 and our observation following Definition 6.1 imply the following result.

Corollary 6.5 For any game $v, L_{1}(v) \subseteq E P(v) \subseteq U D(v)$.

Lemma 4.8 and Theorem 6.4 imply:

Corollary 6.6 For any game $v$, the set $\operatorname{EP}(v)$ is compact.

Corollary 6.5 and Theorem 5.5 imply that the $L_{1}$-center, the excess Pareto optimal set and the undominated set coincide for three-person games. The next example shows that in general $E P(v)$ can be a proper subset of $U D(v)$.

Example 6.7 Let $(N, v)$ be defined by $N=\{1,2,3,4\}$ and, for $S \subseteq N, v(S)=$ 1 if $S=\{1\}$ or $|S|=2, v(N)=v(\emptyset)=0$, and $v(S)=-1$ for all other coalitions in $N$. We claim that $x=0 \in \mathbb{R}^{N}$ is an element of $U D(v)$. Indeed, $L(x)$ consists of coalition $\{1\}$ and all 2-person coalitions; $E(x)=\{\emptyset, N\}$; and $H(x)$ consists of all other coalitions. So $L(x)$ and $H(x)$ are balanced and therefore $x \in U D(v)$ by Theorem 4.2. However, $L H(x)=\{1\}$, which is not balanced within $\operatorname{LEH}(x)=\{\emptyset, 1, N\}$, so that $x \notin E P(v)$ by Theorem 6.3 .

Like $U D(v)$ the set $E P(v)$ is not necessarily convex, as the following example shows. This also implies that $L_{1}(v)$ can be a proper subset of $E P(v)$. 
Example 6.8 Let $N=\{1, \ldots, 4\}$ and let $v$ be defined by

$$
\begin{aligned}
& v(13)=v(14)=1, v(N)=v(\emptyset)=0, v(134)=-2, \\
& v(12), v(234)=14, \text { and } v(S)=-14, \text { otherwise. }
\end{aligned}
$$

Let $x=(0, \ldots, 0)$ and $x^{\prime}=(9,3,-6,-6)$. As $L(x)$ consists of $\{1,2\},\{1,3\}$, $\{1,4\}$, and $\{2,3,4\}, x \in E P(v)$ by Theorem 6.3. Similarly, $L\left(x^{\prime}\right)=\{12,134$, $234\}$ is balanced so that $x^{\prime} \in E P(v)$. Now, let $z=x^{\prime} / 2$. Then $L(z)=\{12,234\}$ and $E(z)=\{\emptyset, N\}$ so that $z \notin E P(v)$.

In Section 7 we will show that although $E P(v)$ is not necessarily convex, it is contractible and in particular connected.

We proceed with an interesting class of games for which the undominated set and the excess Pareto optimal set coincide. First, recall that the dual of a game $(N, v)$ is the game $\left(N, v^{*}\right)$, defined by $v^{*}(S)=v(N)-v(N \backslash S)$ for all $S \subseteq N$. Of course, $v^{* *}=v$. Further, for games $(N, v)$ and $(N, w)$ denote by $(N, v \wedge w)$ the coalition-wise minimum of $v$ and $w$, i.e., $(v \wedge w)(S)=\min \{v(S), w(S)\}$ for all $S \subseteq N$. We start with the following lemma.

Lemma 6.9 For any game $v, E P(v)=E P\left(v \wedge v^{*}\right)$.

Proof. Let $v$ be an arbitrary game and write $w=v \wedge v^{*}$. It is straightforward to check that for every $S \subseteq N$ we have

$$
\begin{aligned}
& w(S)=v(S) \text { and } w(N \backslash S)=v(N \backslash S) \quad \Leftrightarrow \quad v(S)+v(N \backslash S) \leqslant v(N) \\
& w(S)=v^{*}(S) \text { and } w(N \backslash S)=v^{*}(N \backslash S) \Leftrightarrow v(S)+v(N \backslash S) \geqslant v(N) \text {. }
\end{aligned}
$$

Moreover, $v(N)=w(N)$ and therefore $X(v)=X(w)$. Hence, if $S \subseteq N$ and $v(S)+v(N \backslash S) \leqslant v(N)$, then $f(S, x, v)=f(S, x, w)$. If $S \subseteq N$ and $v(S)+$ $v(N \backslash S) \geqslant v(N)$, then $f(S, x, v)=|v(S)-x(S)|+|v(N \backslash S)-x(N \backslash S)|=$ $\left|x(N \backslash S)-v^{*}(N \backslash S)\right|+\left|x(S)-v^{*}(S)\right|=f(S, x, w)$. Hence, $E P(v)=E P(w)=$ $E P\left(v \wedge v^{*}\right)$.

The following definition presents weakenings of the familiar superadditivity and subadditivity conditions.

Definition 6.10 A game $v$ is proper if $v(S)+v(N \backslash S) \leqslant v(N)$ for all $S \subseteq N$, and antiproper if $v(S)+v(N \backslash S) \geqslant v(N)$ for all $S \subseteq N .^{5}$

Lemma 6.11 For any game $v$, the game $v \wedge v^{*}$ is proper. Moreover, $v$ is proper if and only if $v=v \wedge v^{*}$.

Proof. Let $v$ be a game and $S$ an arbitrary coalition. Then $\left(v \wedge v^{*}\right)(S)+(v \wedge$ $\left.v^{*}\right)(N \backslash S)=\min \{v(S), v(N)-v(N \backslash S)\}+\min \{v(N \backslash S), v(N)-v(S)\}$. If $v(S)+v(N \backslash S) \leqslant v(N)$, then this expression is equal to $v(S)+v(N \backslash S)$, and otherwise it is equal to $2 v(N)-v(S)-v(N \backslash S)$. In the first case, $\left(v \wedge v^{*}\right)(S)+(v \wedge$

\footnotetext{
${ }^{5}$ The word 'proper' is usually employed for simple games, with the same meaning.
} 
$\left.v^{*}\right)(N \backslash S) \leqslant v(N)=\left(v \wedge v^{*}\right)(N)$; in the second case, $\left(v \wedge v^{*}\right)(S)+\left(v \wedge v^{*}\right)(N \backslash S)=$ $2 v(N)-v(S)-v(N \backslash S) \leqslant v(N)=\left(v \wedge v^{*}\right)(N)$. Hence $v \wedge v^{*}$ is proper.

Of the second statement we only still have to show the only-if statement, but this follows from (6).

Lemma 6.12 For any game $v, E P(v)=U D\left(v \wedge v^{*}\right)$.

Proof. Let $v$ be an arbitrary game and write $w=v \wedge v^{*}$. By Lemma 6.9 and Corollary 6.5 it is sufficient to show that $U D(w) \subseteq E P(w)$. Let $x \in U D(w)$. By Lemma 6.11, $w(S)+w(N \backslash S) \leqslant w(N)$ for all $S \subseteq N$. Hence $L H(x, w)=$ $L(x, w)$ and $L E H(x, w)=L(x, w) \cup E(x, w)$. Hence $L H(x, w)$ is balanced within $\operatorname{LEH}(x, w)$ by Theorem 4.2 , and thus $x \in E P(w)$ by Theorem 6.3 .

It is straightforward to check that both $E P$ and $U D$ are self-dual, i.e., $E P(v)=E P\left(v^{*}\right)$ and $U D(v)=U D\left(v^{*}\right)$.

Corollary 6.13 For any proper or antiproper game $v, E P(v)=U D(v)$.

Proof. If $v$ is proper the corollary follows from Lemmas 6.11 and 6.12. If $v$ is antiproper then $v^{*}$ is proper so that $E P\left(v^{*}\right)=U D\left(v^{*}\right)$ by the same lemmas. Now $E P(v)=U D(v)$ follows by self-duality of $E P$ and $U D$.

We conclude with the following theorem, which says that any efficient allocation not in $E P(v)$ is excess Pareto dominated by an allocation in $E P(v)$. It shows that in a specific sense $E P(v)$ is the Pareto optimal set of $X(v)$.

Theorem 6.14 Let $v$ be a game and let $x \in X(V) \backslash E P(v)$. Then $D(x) \cap$ $E P(v) \neq \emptyset$.

Proof. Define the set $\bar{D}(x)$ by $\bar{D}(x)=\{z \in X(v) \mid f(z) \leqslant f(x)\}$. It can be checked that $z \in \bar{D}(x)$ if and only if $|v(N)+v(S)-v(N \backslash S)-2 z(S)| \leqslant f(S, x)$ for all $S \subseteq N$. This implies in particular that $\bar{D}(x)$ is a nonempty polytope. Since the map $\ell$ is continuous we have

$$
\emptyset \neq D^{m}(x):=\left\{z \in \bar{D}(x) \mid \ell(z) \leqslant \ell\left(z^{\prime}\right) \text { for all } z^{\prime} \in \bar{D}(x)\right\} .
$$

Take any $z \in D^{m}(x)$, then clearly $D(z)=\emptyset$ and $z \in D(x)$. Hence, $z \in$ $E P(v) \cap D(x)$.

\section{Contractibility of the set of excess Pareto op- timal allocations}

We have already seen that for a game $v$ neither the set of excess Pareto optimal allocations $E P(v)$ nor the undominated set $U D(v)$ have to be convex. In this section we show that $E P(v)$ satisfies the weaker condition of contractibility, implying, in particular that it is connected. It follows (cf. Corollary 6.13) that 
if $v$ is proper or antiproper then also $U D(v)$ is contractible and thus connected. Contractibility or connectedness of $U D(v)$ in general is left as an open problem.

For a game $v$ define $T^{v}=\left\{f(x) \in \mathbb{R}^{2^{N}} \mid x \in E P(v)\right\}$ and

$$
T_{+}^{v}=T^{v}+\mathbb{R}_{+}^{2^{N}}=\left\{t \in \mathbb{R}^{2^{N}} \mid t \geqslant t^{\prime} \text { for some } t^{\prime} \in T^{v}\right\} .
$$

Lemma 7.1 Let $v$ be a game. Then $T_{+}^{v}$ is nonempty, closed, and convex.

Proof. Nonemptiness of $T_{+}^{v}$ is obvious, and convexity follows from convexity of the functions $f(S, \cdot)$. For closedness, let $t^{1}, t^{2}, \ldots \in T_{+}^{v}$ converge to $t \in \mathbb{R}^{2^{N}}$ and $x^{k} \in E P(v)$ with $f\left(x^{k}\right) \leqslant t^{k}$ for each $k \in \mathbb{N}$. Since $E P(v)$ is compact (Corollary 6.6), we may assume that $x^{1}, x^{2}, \ldots$ converges to some $x \in \operatorname{EP}(v)$. By continuity of $f(\cdot), f(x) \leqslant t$ and thus $t \in T_{+}^{v}$.

Recall that a set $X \subseteq \mathbb{R}^{m}$ is contractible if there exists a continuous map $g:[0,1] \times X \rightarrow X$ and a point $p \in X$ such that $g(0, x)=x$ and $g(1, x)=p$ for all $x \in X{ }^{6}$ Peleg (1972, Theorem 4.6) shows that the set of Pareto optimal elements of any closed convex subset of some Euclidean space is contractible. Clearly, this result also applies with Pareto optimality used in our sense of 'Pareto minimality'. Hence, with Lemma 7.1 we obtain the following result.

Lemma 7.2 For any game $v$ the set $T^{v}$ is contractible.

For $t \in T^{v}$ we consider the inverse image $f^{-1}(t, v)=f^{-1}(t)=\{x \in X(v) \mid$ $f(x)=t\}$. By definition, $f^{-1}(t) \subseteq E P(v)$. If $x \in f^{-1}(t)$ and $z \in X(v)$ then $z \in f^{-1}(t)$ if and only if for all $S \subseteq N$

$$
\begin{array}{lll}
S \in L(x) \text { and } N \backslash S \in H(x) & \Rightarrow & z(S)=x(S) \\
S, N \backslash S \in L(x) \cup E(x) & \Leftrightarrow & S, N \backslash S \in L(z) \cup E(z) \\
S, N \backslash S \in H(x) \cup E(x) & \Leftrightarrow & S, N \backslash S \in H(z) \cup E(z) .
\end{array}
$$

This implies that $f^{-1}(t)$ is a convex polytope for each $t \in T^{v}$. Thus, $f^{-1}(\cdot)$ is a correspondence mapping elements of $T^{v}$ to convex compact subsets of $\operatorname{EP}(v)$. We now show that this correspondence is continuous, i.e., upper hemicontinuous (uhc) and lower hemicontinuous (lhc).

Lemma 7.3 The correspondence $f^{-1}(\cdot): T^{v} \rightarrow E P(v)$ is continuous.

Proof. In order to show uhc of $f^{-1}(\cdot)$, let $t^{1}, t^{2}, \ldots, t \in T^{v}$ with $t^{k} \rightarrow t$ and let $x^{k} \in f^{-1}\left(t^{k}\right)$ for each $k \in \mathbb{N}$ such that $x^{k} \rightarrow x \in E P(v)$. Then by continuity of $f$, we have $f(x)=t$, and hence $x \in f^{-1}(t)$.

For lhc, let again $t^{1}, t^{2}, \ldots, t \in T^{v}$ with $t^{k} \rightarrow t$ and let $z \in f^{-1}(t)$. We have to show that there are $z^{k} \in f^{-1}\left(t^{k}\right)$ for $k=1,2, \ldots$ with $z^{k} \rightarrow z$. We take, for each $k, z^{k} \in f^{-1}\left(t^{k}\right)$ such that $\left|z^{k}-z\right|$ (Euclidean distance) is minimal: these points $z^{k}$ exist and are unique since $f^{-1}\left(t^{k}\right)$ is a compact convex set. Since all these points $z^{k}$ are in the compact set $\operatorname{EP}(v)$, there is a converging

\footnotetext{
${ }^{6}$ I.e., the set $X$ is homotopic to $p$ via the homotopy $g$.
} 
subsequence, say $z^{1}, z^{2}, \ldots$ itself, with limit some $\tilde{z} \in E P(v)$. It is sufficient to show that $\tilde{z}=z$. Note that $\tilde{z} \in f^{-1}(t)$ by continuity of $f$. Since $z^{k} \rightarrow \tilde{z}$ we may assume without loss of generality that there is a $\delta>0$ such that for all $S \subseteq N$ we have

$$
\begin{aligned}
& S \in L(\tilde{z}) \quad \Rightarrow \quad z^{k}(S)<v(S)-\delta \text { for all } k \\
& S \in H(\tilde{z}) \quad \Rightarrow \quad z^{k}(S)>v(S)+\delta \text { for all } k .
\end{aligned}
$$

We assume that $\tilde{z} \neq z$ and derive a contradiction. Since $z^{k} \rightarrow \tilde{z} \neq z$ we may assume without loss of generality that

$$
\left|z^{k}-\tilde{z}\right|<\left|z^{k}-z\right| \text { for all } k \text {. }
$$

For $\varepsilon>0$ and each $k$ we define $z^{k, \varepsilon}=z^{k}+\varepsilon(z-\tilde{z})$. Then

$$
\begin{aligned}
\left|z^{k, \varepsilon}-z\right| & =\left|(1-\varepsilon) z^{k}-(1-\varepsilon) z+\varepsilon\left(z^{k}-\tilde{z}\right)\right| \\
& \leqslant(1-\varepsilon)\left|z^{k}-z\right|+\varepsilon\left|z^{k}-\tilde{z}\right| \\
& <\left|z^{k}-z\right|
\end{aligned}
$$

where the final inequality follows from (9). Hence, by the choice of $z^{k}$, it follows that $z^{k, \varepsilon} \notin f^{-1}\left(t^{k}\right)$ for all $k$ and $\varepsilon>0$. Choose $\varepsilon>0$ so small that $\varepsilon(z(S)-$ $\tilde{z}(S))<\delta$ for all $S \subseteq N$. We will now show that then $f\left(z^{k, \varepsilon}\right) \leqslant f\left(z^{k}\right)$ for all $k$, contradicting $z^{\bar{k}, \varepsilon} \notin f^{-1}\left(t^{k}\right)$ in case $f\left(z^{k, \varepsilon}\right)=f\left(z^{k}\right)$ and contradicting $z^{k} \in E P(v)$ in case $f\left(z^{k, \varepsilon}\right) \leqslant f\left(z^{k}\right), f\left(z^{k, \varepsilon}\right) \neq f\left(z^{k}\right)$.

In order to show $f\left(z^{k, \varepsilon}\right) \leqslant f\left(z^{k}\right)$ we distinguish the following four cases (the remaining cases are analogous): (a) $S \in L(\tilde{z}), N \backslash S \in H(\tilde{z})$; (b) $S \in L(\tilde{z})$, $N \backslash S \in L(\tilde{z}) ;(\mathrm{c}) S \in L(\tilde{z}), N \backslash S \in E(\tilde{z})$; and (d) $S, N \backslash S \in E(\tilde{z})$.

Case (a) $S \in L(\tilde{z}), N \backslash S \in H(\tilde{z})$. In this case, by $(7), \tilde{z}(S)=z(S)$, hence $f\left(S, z^{k, \varepsilon}\right)=f\left(S, z^{k}\right)$.

Case (b) $S \in L(\tilde{z}), N \backslash S \in L(\tilde{z})$. In this case, by $(8), z^{k}(S)<v(S)-\delta$ and $z^{k}(N \backslash S)<v(N \backslash S)-\delta$. Since $\varepsilon(z(T)-\tilde{z}(T))<\delta$ for all $T \subseteq N$ we obtain $z^{k, \varepsilon}(S)<v(S)$ and $z^{k, \varepsilon}(N \backslash S)<v(N \backslash S)$, so that again $f\left(S, z^{k, \varepsilon}\right)=f\left(S, z^{k}\right)$.

Case (c) $S \in L(\tilde{z}), N \backslash S \in E(\tilde{z})$. In this case, by (7), $S, N \backslash S \in L(z) \cup E(z)$, hence $z^{k, \varepsilon}(N \backslash S)=z^{k}(N \backslash S)+\varepsilon(z(N \backslash S)-\tilde{z}(N \backslash S)) \leqslant z^{k}(N \backslash S)$ and thus $z^{k, \varepsilon}(S) \geqslant z^{k}(S)$. Since, by $(8), z^{k}(S)<v(S)-\delta$ and thus, by $\varepsilon(z(T)-\tilde{z}(T))<\delta$, $z^{k, \varepsilon}(S)<v(S)$, we conclude that $f\left(S, z^{k, \varepsilon}\right) \leqslant f\left(S, z^{k}\right)$.

Case (d) $S, N \backslash S \in E(\tilde{z})$. In this case, by (7), $S, N \backslash S \in L(z) \cup E(z)$ or $S, N \backslash S \in H(z) \cup E(z)$. We assume the former, the argument for the latter is analogous. Then $z^{k, \varepsilon}(T)=z^{k}(T)+\varepsilon(z(T)-\tilde{z}(T)) \leqslant z^{k}(S)$ for both $T=S$ and $T=N \backslash S$, so that we have $z^{k, \varepsilon}(T)=z^{k}(T)$ for both $T=S$ and $T=N \backslash S$, and thus $f\left(S, z^{k, \varepsilon}\right)=f\left(S, z^{k}\right)$.

Theorem 7.4 For any game $v$ the set $\operatorname{EP}(v)$ is contractible.

Proof. For every $t \in T^{v}$ let $x^{t}$ be the lexicographically maximal element of $f^{-1}(t)$. Let $E P^{\operatorname{lex}}(v)=\left\{x^{t} \mid t \in T^{v}\right\}$. The map $f: E P^{\operatorname{lex}}(v) \rightarrow T^{v}$, i.e., the 
restriction of $f$ to $E P^{\operatorname{lex}}(v)$, is a continuous bijection. Also its inverse, denoted by $f^{-1}$, is continuous, which can be seen as follows. Let $t^{1}, t^{2}, \ldots, t \in T^{v}$ with $t^{k} \rightarrow t$. We have to show that $f^{-1}\left(t^{k}\right) \rightarrow f^{-1}(t)$, i.e., that $x^{t^{k}} \rightarrow x^{t}$. Since $x^{t^{k}}$ is an element of the bounded (even compact) set $\operatorname{EP}(v)$ for each $k$, there is a converging subsequence, and it is sufficient to prove that this subsequence has limit $x^{t}$. For simplicity of notation let $\left(x^{t^{k}}\right)_{k \in \mathbb{N}}$ be this subsequence. By Lemma 7.3 (specifically, by lhc of $\left.f^{-1}(\cdot)\right)$ there is a sequence $\left(x^{k}\right)_{k \in \mathbb{N}}$ with $x^{k} \in f^{-1}\left(t^{k}\right)$ and $x^{k} \rightarrow x^{t}$. Since $x^{t^{k}}$ is the lexicographic maximum of $f^{-1}\left(t^{k}\right)$ for each $k$ and $x^{t}$ is the lexicographic maximum of $f^{-1}(t)$, we have $x^{t^{k}} \rightarrow x^{t}$.

Thus, $f$ is a homeomorphism between $E P^{\operatorname{lex}}(v)$ and $T^{v}$ and therefore, by Lemma 7.2, $E P^{\text {lex }}(v)$ is contractible. This implies that there is an $\hat{x} \in E P^{\text {lex }}(v)$ and a continuous function $g:[0,1] \times E P^{\operatorname{lex}}(v) \rightarrow E P^{\operatorname{lex}}(v)$ such that $g(0, x)=x$ and $g(1, x)=\hat{x}$ for all $x \in E P^{\text {lex }}(v)$. Define the function $h:[0,1] \times E P(v) \rightarrow$ $E P(v)$ by

$$
h(\alpha, x)= \begin{cases}(1-2 \alpha) x+2 \alpha x^{f(x)} & \text { if } 0 \leqslant \alpha \leqslant \frac{1}{2} \\ g\left(2 \alpha-1, x^{f(x)}\right) & \text { if } \frac{1}{2} \leqslant \alpha \leqslant 1\end{cases}
$$

for all $(\alpha, x) \in[0,1] \times E P(v)$. Then, by convexity of the set $f^{-1}(t)$, we have $h(\alpha, x) \in f^{-1}(t)$ for all $x \in E P(v), t=f(x)$, and $0 \leqslant \alpha \leqslant \frac{1}{2}$; and $h$ is continuous, in particular, since $g$ is. Also, $h(0, x)=x$ and $h(1, x)=\hat{x}$ for all $x \in E P(v)$. Thus, $\operatorname{EP}(v)$ is contractible.

\section{The min-prenucleolus}

An interesting question is whether there exists a single-valued solution for TUgames which always assigns a point in $U D(v)$ or $E P(v)$. An obvious candidate for such a solution is the prenucleolus (Schmeidler, 1969), since this is contained in the core if the core is not empty. It is defined as follows. Recall that for a game $(N, v)$, a coalition $S$ and an allocation $x \in X(v), e(S, x, v)=v(S)-x(S)$ denotes the excess of $S$ at $x$. By $\theta(x) \in \mathbb{R}^{2^{|N|}-2}$ we denote the vector in which the excesses of all nonempty proper coalitions in $N$ are arranged in nonincreasing order. The prenucleolus of $(N, v)$ is the efficient allocation, denoted $\nu(N, v)=$ $\nu(v)$, which lexicographically minimizes $\theta(x)$ over all $x \in X(v)$.

The following example, however, shows that even in three-player games, where the undominated set, the excess Pareto optimal set, and the $L_{1}$-center coincide, the prenucleolus does not have to be in the undominated set.

Example 8.1 Consider the three-player game $v$ with $v(1)=v(2)=v(3)=$ $v(23)=1, v(12)=v(13)=-1$, and $v(123)=0$. The prenucleolus of this game is $x=(0,0,0)$ but $E(x, v)=\{N, \emptyset\}$ and $H(x, v)=\{12,13\}$, so that by Theorem 4.2 we have $x \notin U D(v)$.

We now propose a modification of the prenucleolus and prove that this solution always assigns a point in $E P(v)$ and hence in $U D(v)$. 
Definition 8.2 The min-prenucleolus of $v$, denoted by $\nu_{\wedge}(v)$, is defined by $\nu_{\wedge}(v)=\nu\left(v \wedge v^{*}\right)$

If the game $v$ is proper then by Lemma 6.11 the min-prenucleolus of $v$ is just the prenucleolus of $v$. The game in Example 8.1 is not proper, and as the following example shows its min-prenucleolus is in the undominated set.

Example 8.3 Consider the three-player game $v$ from Example 8.1. Then $v^{*}$ is given by $v^{*}(2)=v^{*}(3)=1, v^{*}(1)=v(12)=v(13)=v^{*}(23)=-1$, and $v^{*}(123)=0$. Then the game $w=v \wedge v^{*}$ is equal to $v^{*}$ and has minprenucleolus equal to $x=\left(-\frac{4}{3}, \frac{2}{3}, \frac{2}{3}\right)$. Now $L(x, v)=\{1,2,3\}, E(x, v)=\{\emptyset, N\}$, and $H(x, v)=\{12,13,23\}$, so that $x \in U D(v)$ by Theorem 4.2.

Proposition 8.4 For any game $v, \nu_{\wedge}(v) \in E P(v)$.

Proof. Let $w=v \wedge v^{*}$ and $x=\nu(w)$. By Lemma 6.9 it is sufficient to show that $x \in E P(w)$. As $w(S)+w(N \backslash S) \leqslant w(N), S \in L(x, w)$ implies $N \backslash S \in$ $H(x, w)$, i.e., $L H(x, w)=L(x, w)$. By the characterization of the prenucleolus by Kohlberg (1971), $\{N\} \cup L(x, w)$ is balanced so that the proof is complete by Theorem 6.3.

The min-prenucleolus does not have to be in the $L_{1}$-center, as the following example shows.

Example 8.5 Let $v$ be the four-person game defined in Example 6.8. For this game $L_{1}(v) \neq E P(v)$ and the game is proper so that its min-prenucleolus is equal to its prenucleolus $x=\left(-5 \frac{1}{5}, 10 \frac{2}{5},-2 \frac{3}{5},-2 \frac{3}{5}\right)$. Then one can check that $\ell(x)>\ell(0,0,0,0)$, so that $x \notin L_{1}(v)$.

We present an axiomatization of the min-prenucleolus based on a reduced game property, among other axioms. To this end we make the set of players variable. The universe of potential players is $U \subseteq \mathbb{N}$, with $\{1,2,3,4\} \subseteq U$. A game is now any pair $(N, v)$ such that $\emptyset \neq N \subseteq U$ is finite and $v: 2^{N} \rightarrow \mathbb{R}$, $v(\emptyset)=0$. The set of all games is denoted by $\Gamma_{U}$.

Let $\sigma$ be a solution, i.e., $\sigma(N, v) \subseteq\left\{x \in \mathbb{R}^{N} \mid x(N) \leqslant v(N)\right\}$ for all $(N, v) \in$ $\Gamma_{U}$. Then $\sigma$ satisfies

(i) single-valuedness (SIVA) if $|\sigma(N, v)|=1$ for all $(N, v) \in \Gamma_{U} ;^{7}$

(ii) Pareto optimality (PO) if $\sigma(N, v) \subseteq X(N, v)$ for all $(N, v) \in \Gamma_{U}$;

(iii) anonymity (AN) if, for any $(N, v) \in \Gamma_{U}$ and any injection $\tau: N \rightarrow U$, $\sigma(\tau(N), \tau v)=\tau(\sigma(N, v))$, where $\tau v(S)=v\left(\tau^{-1}(S)\right)$ for all $S \subseteq \tau(N)$, and $\tau(x)_{i}=x_{\tau^{-1}(i)}$ for all $i \in \tau(N)$ and $x \in \mathbb{R}^{N}$;

(iv) covariance under strategic equivalence $(\mathrm{COV})$ if $\sigma(N, a v+b)=a \sigma(N, v)+b$ for any $(N, v) \in \Gamma_{U}, a>0, b \in \mathbb{R}^{N}$;

\footnotetext{
${ }^{7}$ In case $\sigma$ is single-valued we identify $\sigma(N, v)$ with its unique element.
} 
(v) the reduced game property (RGP) if, for $(N, v) \in \Gamma_{U}$ and $\emptyset \neq S \subseteq N$, $x_{S} \in \sigma\left(S, v^{S, x}\right)$ for all $x \in \sigma(N, v)$, where $x_{S}:=\left(x_{i}\right)_{i \in S}$ and where the reduced game $\left(S, v^{S, x}\right)$ is defined by

$$
v^{S, x}(T)= \begin{cases}v(N)-x(N \backslash S) & \text { if } T=N, \\ \max _{Q \subseteq N \backslash S} v(T \cup Q)-x(Q) & \text { if } \emptyset \neq T \mp S, \\ 0 & \text { if } T=\emptyset .\end{cases}
$$

(vi) self-duality (SD) if $\sigma(N, v)=\sigma\left(N, v^{*}\right)$ for all $(N, v) \in \Gamma_{U}$.

Remark 8.6 Sobolev (1975) showed that the prenucleolus is the unique solution that satisfies SIVA, COV, AN and RGP, provided that $|U|=\infty$.

Our solution $\nu_{\wedge}$ satisfies all of the foregoing properties except RGP. We weaken this property, as follows. A solution $\sigma$ satisfies

(viii) the min-reduced game property (min-RGP) if, for $(N, v) \in \Gamma_{U}, \emptyset \neq S \subseteq N$, and $x \in \sigma(N, v)$ we have: if $v^{S, x}=v^{S, x} \wedge\left(v^{S, x}\right)^{*}$ (i.e., if $v^{S, x}$ is proper), then $x_{S} \in \sigma\left(S, v^{S, x}\right)$.

Moreover, we need the following property, which is stronger than SD. A solution $\sigma$ satisfies

(ix) min-self-duality (min-SD) if $\sigma\left(N, v \wedge v^{*}\right)=\sigma(N, v)$ for all $(N, v) \in \Gamma_{U}$.

Observe that, indeed, min-SD implies SD: for a game $v$, we have $\sigma(N, v)=$ $\sigma\left(N, v \wedge v^{*}\right)=\sigma\left(N, v^{*} \wedge v\right)=\sigma\left(N, v^{*}\right)$ since $v^{* *}=v$.

Now, $\nu_{\wedge}$ can be characterized as follows.

Theorem 8.7 The solution $\nu_{\wedge}$ is the unique solution that satisfies SIVA, COV, $\mathrm{AN}$, min-RGP, and min-SD, provided that $|U|=\infty$.

Proof. Let $(N, v) \in \Gamma_{U}$, let $\tau: N \rightarrow U$ be an injection, $b \in \mathbb{R}^{N}, a>0$, and $w=v \wedge v^{*}$. Then $\tau w=\tau v \wedge \tau v^{*}$ and $a w+b=(a v+b) \wedge(a v+b)^{*}$. Hence $\nu_{\wedge}$ satisfies SIVA, COV, and AN because $\nu$ satisfies these properties (see Remark 8.6), and it satisfies min-SD because $w \wedge w^{*}=w$. In order to show that $\nu_{\wedge}$ satisfies min-RGP, let $x=\nu(N, w)$ and $\emptyset \neq S \subseteq N$ such that $u=u \wedge u^{*}$, where $u=v^{S, x}$. Since the prenucleolus satisfies RGP, it suffices to show that $u=w^{S, x}$. As $w \leqslant v$, we have $w^{S, x} \leqslant u$. Let $T \subseteq S$. It remains to show that $u(T) \leqslant w^{S, x}(T)$. If $T=\emptyset$ then $w^{S, x}(T)=0=u(T)$. If $T=S$ then $u(T)=v(N)-x(N \backslash S)=w(N)-x(N \backslash S)=w^{S, x}(T)$ since $v(N)=v^{*}(N)$. If $\emptyset \neq T \mp S$, then there exists $Q \subseteq N \backslash S$ such that $u(T)=v(T \cup Q)-x(Q)$. Let $R=(N \backslash S) \backslash Q$. Then $u(S \backslash T) \geqslant v((S \backslash T) \cup R)-x(R)$. Since $u(T)+u(S \backslash T) \leqslant$ $u(S)=v(N)-x(N \backslash S)$, we have

$$
v(T \cup Q)-x(Q)+v((S \backslash T) \cup R)-x(R) \leqslant v(N)-x(N \backslash S)
$$


hence $v(T \cup Q) \leqslant v(N)-v((S \backslash T) \cup R)$. Then

$$
\begin{aligned}
w(T \cup Q) & =\min \{v(T \cup Q), v(N)-v((S \backslash T) \cup R)\} \\
& =v(T \cup Q) .
\end{aligned}
$$

Thus,

$$
\begin{aligned}
w^{S, x}(T) & =\max _{P \subseteq N \backslash S}\{w(T \cup P)-x(P)\} \\
& \geqslant w(T \cup Q)-x(Q) \\
& =v(T \cup Q)-x(Q) \\
& =u(T) .
\end{aligned}
$$

In order to show uniqueness, let $\sigma$ be a solution that satisfies the five axioms. We show first that $\sigma$ satisfies PO. Let $(N, v) \in \Gamma_{U}$ and $x=\sigma(N, v)$. If $|N|=1$, i.e., $N=\{i\}$ for some $i \in U$, then $x_{i} \leqslant v(\{i\})$. By COV we may assume $v(\{i\})=0$. If we had $x_{i}<0$, then $2 x_{i} \neq x_{i}$ whereas $v=2 v$, so that COV is violated. Hence, $x_{i}=0$. If $|N| \geqslant 2$, then for any $i \in N,\left(\{i\}, v^{\{i\}, x}\right)$ coincides with its dual so that, by min-RGP, $x_{i}=v^{\{i\}, x}(\{i\})=v(N)-x(N \backslash\{i\})$. Hence, $x(N)=v(N)$.

Now we are able to finish the proof. Let $(N, v) \in \Gamma_{U}$ and $x=\nu_{\wedge}(N, v)$. In order to show that $x=\sigma(N, v)$, by min-SD and COV we may assume that $v=v \wedge v^{*}$ and $x=0 \in \mathbb{R}^{N}$. Hence, in particular, $0=\nu(N, v)$. According to Sobolev (1975) there exists a game $(M, u) \in \Gamma_{U}$ with the following properties: (1) $N \subseteq M$; (2) $u(N)=0$; (3) for all $i, j \in M$ there exists a permutation $\tau: M \rightarrow M$ such that $\tau u=u$ and $\tau(i)=j$; and (4) with $y=0 \in \mathbb{R}^{M}, u^{N, y}=v$. Then, by PO and AN, $\sigma(M, u)=y$. By min-RGP, $y_{S}=0=x=\sigma(N, v)$.

We conclude this section with a few remarks.

Remark 8.8 A careful inspection of Sobolev's (1975) proof shows that the game $(M, u)$ in the proof of Theorem 8.7 is proper. Hence, on the set of proper games the prenucleolus is characterized by SIVA, COV, AN, and min-RGP, provided that $|U|=\infty$. This suggests that a characterization of the prenucleolus on other classes of games may be obtained by modifying the axioms in an appropriate way. In particular, RGP should be restricted to hold for those reduced games that are in the class of games under consideration.

Remark 8.9 We show that each of the axioms in Theorem 8.7 is logically independent of the remaining axioms. Define, for any $(N, v) \in \Gamma_{U}, \sigma^{1}(N, v)=$ $X(N, v), \sigma^{2}(N, v)=x$ to be the "equal split solution", i.e., $x_{i}=v(N) /|N|$ for all $i \in N, \sigma^{4}(N, v)=\nu(N, v), \sigma^{5}(N, v)$ to be the Shapley value of $v \wedge v^{*}$. Then $\sigma^{j}, j=1,2,4,5$ satisfies all axioms except the $j$-th one. We recall that

$$
C_{+}(N, v)=\left\{x \in X(N, v) \mid v(S)-x(S) \leqslant(v(S)-\nu(S))_{+}\right\},
$$

where $t_{+}=\max \{0, t\}$ for $t \in \mathbb{R}$ and $\nu=\nu(N, v)$, is called the positive core of $(N, v)$ (see Orshan and Sudhölter, 2010). Select a total order $\succeq$ on $U$ and define

$$
\sigma^{3}(N, v)=\left\{x \in C_{+}\left(N, v \wedge v^{*}\right) \mid x \succeq_{\text {lex }} y \text { for all } y \in C\left(N, v \wedge v^{*}\right)\right\}
$$


where $\succeq_{\text {lex }}$ is the lexicographic order induced by $\succeq$, i.e., if $x, y \in \mathbb{R}^{N}$, then $x \succeq_{\text {lex }} y$ is defined by

$$
i \in N, y_{i}>x_{i} \Longrightarrow \text { there exists } j \in N \text { with } x_{j}>y_{j} \text { and } j \succeq i .
$$

Similarly to Lemma 6.3.15 of Peleg and Sudhölter (2007) it may be shown that $\sigma^{3}$ satisfies SIVA, COV, min-RGP, min-SD, and violates AN. Finally, if $4 \leqslant|U|<\infty$ then there exists a solution that satisfies all five axioms, coincides with $\nu_{\wedge}(N, v)$ for all $(N, v) \in \Gamma_{U}$ with $|N|<|U|$, and does not coincide with $\nu_{\wedge}(U, w)$ for some $(U, w) \in \Gamma_{U}$. For a construction of such a solution see Peleg and Sudhölter (2007, Remark 6.3.3 and Exercises 6.2.3 and 6.3.3).

Remark 8.10 Yanovskaya (1999) proposes the absolute prenucleolus, which is defined as the prenucleolus but then replacing the excesses by their absolute values. Consider the three-person game $v$ with $v(N)=v(123)=0, v(1)=$ $v(2)=v(3)=v(23)=-1$, and $v(12)=v(13)=1$. For this (proper) game the absolute prenucleolus is $x=(0,0,0)$, which however does not belong to $U D(v): L(x)=\{12,13\}$ and $E(x)=\{\emptyset, N\}$, so that $L(x)$ is not balanced within $L(x) \cup E(x)$. So the absolute prenucleolus does not select from $U D$ nor from $E P$.

\section{Proper games}

We have seen that on the interesting class of proper games some of the concepts above simplify. In particular, the undominated set and the excess Pareto optimal set coincide, and the min-prenucleolus is by definition just the prenucleolus, and chooses an allocation from the undominated set.

In this section we reconsider the definition of the undominated set from Section 3, and show that it can be simplified in an appealing manner if we restrict attention to proper games. For a game $v$ call an allocation $x \in X(v)$ payoff-undominated if there is no $z \in X(v)$ such that

$$
\min \{z(S), v(S)\} \geqslant \min \{x(S), v(S)\} \text { for all } S \in 2^{N},
$$

with at least one strict inequality. Let $U(v)$ denote the set of all payoffundominated allocations. It is not difficult to see that $x \in U(v)$ if and only if there is no $z \in X(v)$ with $z(S) \geqslant x(S)$ for all $S \in L(x)$, at least one inequality strict, and $z(S) \geqslant v(S)$ for all $S \in E(x) \cup H(x)$. In other words, it is not possible to make all coalitions that receive less than their worth in $x$ at least as well off and some strictly better off, while giving all other coalitions still at least their worth. From the proof of Theorem 4.2 it follows that $x \in U(v)$ if and only if $L(x)$ is balanced within $L(x) \cup E(x)$. Obviously, $U D(v) \subseteq U(v)$ for any game $v$. For proper games the two sets coincide.

Theorem 9.1 Let $v$ be a proper game. Then $U(v)=U D(v)$. 
Proof. Let $x \in U(v)$. It is sufficient to prove that $x \in U D(v)$ and for this it is sufficient to prove that $H(x)$ is balanced within $H(x) \cup E(x)$. We will actually prove that $H(x) \cup E(x)$ is balanced. Let $\mathcal{B}$ be a balanced collection with $L(x) \subseteq \mathcal{B} \subseteq L(x) \cup E(x)$ and let $y$ be a sidepayment with $y(S) \geqslant 0$ for all $S \in H(x) \cup E(x)$. Then for each $S \in \mathcal{B}$ we have $y(S) \leqslant 0$ since $N \backslash S \in$ $H(x) \cup E(x)$ by properness of $v$. Since $\mathcal{B}$ is balanced, $y(S)=0$ for all $S \in \mathcal{B}$ by Lemma 2.1. Since $\mathcal{B} \cup H(x) \cup E(x)=2^{N}$ we have $y(S) \geqslant 0$ for all coalitions $S$, implying $y=0$. Hence, $H(x) \cup E(x)$ is balanced by Lemma 2.1 .

\section{Concluding remarks}

This paper presents an extensive study of four increasing set extensions of the core and the anticore: the $L_{1}$-center, the excess Pareto optimal set, the undominated set, and the set of payoff-undominated allocations. It also presents a single-valued selection from the excess Pareto optimal set, namely the minprenucleolus.

We have focussed on geometric properties of these sets and on balancedness conditions. An interesting avenue for further research is to develop axiomatic characterizations, perhaps by using reduced game properties such as in the characterization of the core by Peleg (1986).

We finally mention that many of our results will go through, with appropriate modifications, if the set of all coalitions $2^{N}$ is replaced by a balanced collection $\mathcal{B}$ containing the empty set and the grand coalition.

\section{Appendix}

Proof of Theorem 5.5. Since the correspondence $U D(\cdot)$ satisfies COV (property (iv) in Section 8) we may normalize the game $v$ such that $v(\{i\})=0$ for each $i \in N$ and $v(N)=1$, assuming that $v(N)>\sum_{i \in N} v(\{i\})$. The proof is analogous in the case $v(N)<\sum_{i \in N} v(\{i\})$, or we may use $U D(-v)=-U D(v)$ in that case. The case $v(N)=0$ will be considered later.

For an $x \in U D(v)$ the collections $L(x, v) \cup E(x, v)$ and $H(x, v) \cup E(x, v)$ each must contain a balanced sub-collection. Writing, respectively, $L E$ and $H E$ for potential candidates, ignoring $N$ and $\emptyset$, and assuming that $v$ is not balanced or antibalanced - these cases are covered by Theorem 4.5 - so that we exclude $L E=\{\emptyset, N\}$ and $H E=\{\emptyset, N\}$, we have the following seven cases, where in each case some necessary conditions are added for the associated polytope to be nonempty:

1. $L E \supseteq\{2,3,12,13\}, H E \supseteq\{1,23\}$; this implies $v(23) \leqslant 0, v(13) \geqslant 1$, $v(12) \geqslant 1$.

2. $L E \supseteq\{1,3,12,23\}, H E \supseteq\{2,13\}$; this implies $v(13) \leqslant 0, v(12) \geqslant 1$, $v(23) \geqslant 1$. 
3. $L E \supseteq\{1,2,13,23\}, H E \supseteq\{3,12\}$; this implies $v(12) \leqslant 0, v(13) \geqslant 1$, $v(23) \geqslant 1$.

4. $H E \supseteq\{2,3,12,13\}, L E \supseteq\{1,23\}$; this implies $v(23) \geqslant 1, v(13) \leqslant 1$, $v(12) \leqslant 1, v(12)+v(13) \leqslant 1$.

5. $H E \supseteq\{1,3,12,23\}, L E \supseteq\{2,13\}$; this implies $v(13) \geqslant 1, v(12) \leqslant 1$, $v(23) \leqslant 1, v(12)+v(23) \leqslant 1$.

6. $H E \supseteq\{1,2,13,23\}, L E \supseteq\{3,12\}$; this implies $v(12) \geqslant 1, v(13) \leqslant 1$, $v(23) \leqslant 1, v(13)+v(23) \leqslant 1$.

7. $L E \supseteq\{12,13,23\}, H E \supseteq\{1,2,3\}$; this implies $v(12) \geqslant 0, v(13) \geqslant 0$, $v(23) \geqslant 0, v(12)+v(13)+v(23) \geqslant 2$.

There is prima facie another case, namely as case 7 but with the roles of $H$ and $L$ swapped, but this is not possible: we cannot have $x(N)=1$ and $x_{i} \leqslant 0$ for $i=1,2,3$. Clearly, in each of these seven cases the set of efficient allocations under consideration forms a polytope in $U D(v)$. We now show that for each instance where two of these cases are not disjoint, the set $U D(v)$ consists again of exactly one polytope. Thus, $U D(v)$ always consists of exactly one polytope, and the theorem (for $v(N)=1$ ) follows from Theorem 5.3 and Corollary 5.4.

Consider case 1 . Clearly, cases 2,3 , and 4 are not compatible with case 1. If cases 1 and 5 are compatible then $v(23) \leqslant 0$ and $v(12)=v(13)=1$; then the game is balanced (with for instance $(1,0,0) \in C(v)$ ), but this was already excluded. If cases 1 and 6 are compatible then $v(13)=1, v(12) \geqslant 1, v(23) \leqslant 0$; also in this case the game is balanced with $(1,0,0) \in C(v)$. If cases 1 and 7 are compatible then $v(23)=0, v(13) \geqslant 1, v(12) \geqslant 1$; again, $(1,0,0) \in C(v)$.

Cases 2 and 3 are analogous to case 1 . Consider case 4 . If cases 4 and 5 are compatible then $v(13)=v(23)=1$ and $v(12) \leqslant 0$; then $(0,0,1) \in C(v)$ and the game is balanced. If cases 4 and 6 are compatible then $v(12)=v(23)=1$ and $v(132) \leqslant 0$; now $(0,1,0) \in C(v)$, so $v$ is balanced. If cases 4 and 7 are compatible then $v(23) \geqslant 1,0 \leqslant v(12), v(13) \leqslant 1$, and $v(12)+v(13) \leqslant 1$; also in this case the game is balanced with $C(v)$ the convex hull of $\{(0, v(12), 1-$ $v(12)),(0,1-v(13), v(13)\}$.

The cases 5 and 6 are analogous to case 4 . This concludes the proof when $v(N)=1$.

We finally consider the case $v(N)=0$, again assuming that the game is not balanced or antibalanced. Now we have eight cases:

1'. $L E \supseteq\{2,3,12,13\}, H E \supseteq\{1,23\}$; this implies $v(23) \leqslant 0, v(13) \geqslant 0$, $v(12) \geqslant 0$.

2'. $L E \supseteq\{1,3,12,23\}, H E \supseteq\{2,13\}$; this implies $v(13) \leqslant 0, v(12) \geqslant 0$, $v(23) \geqslant 0$.

3'. $L E \supseteq\{1,2,13,23\}, H E \supseteq\{3,12\}$; this implies $v(12) \leqslant 0, v(13) \geqslant 0$, $v(23) \geqslant 0$. 
4'. $H E \supseteq\{2,3,12,13\}, L E \supseteq\{1,23\}$; this implies $v(23) \geqslant 0, v(13) \leqslant 0$, $v(12) \leqslant 0$.

5'. $H E \supseteq\{1,3,12,23\}, L E \supseteq\{2,13\}$; this implies $v(13) \geqslant 0, v(12) \leqslant 0$, $v(23) \leqslant 0$.

6'. $H E \supseteq\{1,2,13,23\}, L E \supseteq\{3,12\}$; this implies $v(12) \geqslant 0, v(13) \leqslant 0$, $v(23) \leqslant 0$.

7'. $L E \supseteq\{12,13,23\}, H E \supseteq\{1,2,3\}$; this implies $v(12) \geqslant 0, v(13) \geqslant 0$, $v(23) \geqslant 0$.

8'. $H E \supseteq\{12,13,23\}, L E \supseteq\{1,2,3\}$; this implies $v(12) \leqslant 0, v(13) \leqslant 0$, $v(23) \leqslant 0$.

Like in the first part we consider what happens if two cases are compatible. If cases 1 and 2 or 1 and 3 are compatible then the game has a nonempty anticore. If cases 1 and 4 are compatible then the game is the (additive) zero-game with zero being the unique allocation in the core or anticore. If case 1 is compatible with any of the cases $6-8$ then the zero allocation is in the core. Cases 2 and 3 are analogous to case 1 . If case 4 is compatible with any of the cases 5-8 then again the zero allocation is in the core. Cases 5 and 6 are analogous to case 4 . If, finally, cases 7 and 8 are compatible then $v$ is again the zero-game.

\section{References}

Bejan C, Gómez JC (2009) Core extensions for non-balanced TU-games. International Journal of Game Theory 38:3-16

Bondareva ON (1962) Theory of the core in the $n$-person game. Vestnik Leningradskii Universitet 13:141-142 (in Russian)

Bossert W, Derks J, Peters H (2005) Efficiency in uncertain cooperative games. Mathematical Social Sciences 50:12-23

Derks J, Peters H (1998) Orderings, excess functions, and the nucleolus. Mathematical Social Sciences 36:175-182

Kohlberg E (1971) On the nucleolus of a characteristic function game. SIAM Journal of Applied Mathematics 20:62-66

Maschler M, Peleg B, Shapley LS (1979) Geometric properties of the kernel, nucleolus, and related solution concepts. Mathematics of Operations Research 4:303-383

Orshan G, Sudhölter P (2010) The positive core of a cooperative game. International Journal of Game Theory 39:113-136

Peleg B (1972) Topological properties of the efficient point set. Proceedings of the American Mathematical Society 15:531-535 
Peleg B (1986) On the reduced game property and its converse. International Journal of Game Theory 15:187-200

Peleg B, Sudhölter P (2007) Introduction to the Theory of Cooperative Games. Springer, Berlin Heidelberg, 2nd edition

Schmeidler D (1969) The nucleolus of a characteristic function game. SIAM Journal on Applied Mathematics 17:1163-1170

Shapley LS (1967) On balanced sets and cores. Naval Research Logistics Quarterly 14:453-460

Shapley LS, Shubik M (1966) Quasi-cores in a monetary economy with convex preferences. Econometrica 34:805-827

Sobolev AI (1975) The characterization of optimality principles in cooperative games by functional equations. In: Mathematical Methods in the Social Sciences, edited by N.N. Vorobiev, vol. 6, 95-151, Vilnius, Academy of Sciences of the Lithuanian SSR (in Russian)

Spinetto R (1974) The geometry of solution concepts for $N$-person cooperative games. Management Science 20:1295-1299

Yanovskaya E (1998) Set-valued analogues of the prenucleolus for cooperative TU-games. International Journal on Mathematics, Game Theory and Algebra 7, no. 4

Yanovskaya E (2002) A family of least power values for cooperative games with transferable utilities. In: Tangian A, Gruber J (eds.) Constructing and Applying Objective Functions. Proceedings of the fourth international conference on econometric decision models. Springer, Berlin, 473-494

von Neumann J, Morgenstern O (1944) Theory of Games and Economic Behavior. Princeton University Press, Princeton, NJ 